\title{
Suän, Zetti, Wüer - \\ Namen im Umkreis der Oberwalliser Flurbewässerung
}

\author{
Werner Bellwald (Basel)/Stefan Würth (Berg)
}

\begin{abstract}
An archaic dialect, ancient wooden houses, the special rye bread or the artificial irrigation channels (suones) - they all belong to the typical "sights" of the Valais that are widely known but little investigated. In our paper we record the names of waterways in the Germanspeaking Valais, above all we derive proper names and appellatives etymologically and look into their geografic and diachronic distribution from first documentary evidence back in the 13th century up to present-day oral recordings, from the language boundary near Siders to the Rhone glacier.

Not claiming completeness and not wanting to collect all suones an onomastic explanation of the most important names and appellatives is given which denote artificial waterways. Tools and work processes are listed and a typology and morphology of the names of the waterways is set up.
\end{abstract}

\section{$1 \quad$ Einleitung}

\subsection{Prolog: Die Walliser Suon als Emblem}

Das alemannische Oberwallis ist reich an emblematischen Kulturphänomenen. Dazu zählen der altertümlich wirkende Dialekt, ausgeprägt endogame Gemeindestrukturen, hochgelegene Haufendörfer, lärchenhölzerne Heidenhütten, runenartige Tesselzeichen, das Roggenbrot und auch: die Suonen. Für die wissenschaftliche Erforschung der Walliser Kultur wurden die Grundlagen im Verlauf des 19. und im frühen 20. Jh. gelegt. Zur gleichen Zeit wurden die entsprechenden Phänomene idealistisch aufgeladen. So schuf etwa der Dichter JakobChristoph Heer für die Bewässerungssysteme den Begriff "heilige Wasser". ${ }^{1}$ Das Bild von waghalsig gebauten Holzkänneln an steilen Felswänden fand Eingang in Schulbücher, Landesausstellungen, Bildbände und Filme. Heute werden solche Bilder vor allem vom Tourismus übernommen und in zunehmend unwirklicher und verkürzter Form gepflegt. Derweil fällt es in manchen Fällen schwer, die autochthonen Benennungen von Bewässerungsleitungen noch zu ermitteln.

\section{2 Übersicht}

Ebendiese - die Benennungen der Bewässerungsleitungen - stehen im Zentrum der vorliegenden Arbeit. Vorrangig geht es uns darum, die Namen und Appellativa künstlicher Bewässerungssysteme des deutschsprachigen Oberwallis etymologisch herzuleiten und in ihrer diachronen und geographischen Verteilung zu untersuchen; von den ersten gesicherten urkundlichen Erwähnungen im 13. Jahrhundert bis zur Gegenwart; von der Sprachgrenze oberhalb Siders bis zum Rhonegletscher, in heute 84 Gemeinden, auf 2620 Quadratkilometern. Nicht eine Sammlung von Suonen wird angestrebt, sondern die onomastische Erläute-

\footnotetext{
${ }^{1}$ Der Roman "An heiligen Wassern" von J.C. Heer erfuhr 1905 bereits seine 22. Auflage.
} 
rung der wichtigsten Namen und Appellativa, welche die Wasserleiten bezeichnen. Auch auf die Benennung von Geräten und Tätigkeiten im Rahmen der Flurbewässerung wird eingegangen. Daneben soll eine erste Typologie und Morphologie von den Eigennamen der Oberwalliser Wasserleitungen aufgestellt werden.

Als Quellen dienten die umfangreiche regionale und lokale Literatur, suonenspezifische Arbeiten, publizierte historische Belege und die Dateien der Orts- und Flurnamensammlung Oberwallis. ${ }^{2}$ Daneben erfolgten eigene Erhebungen im Feld.

Eine Suon oder Wasserleite ist ein Kanal von vorwiegend schwacher Neigung, der sein Wasser, das in der Regel von Gletscherbächen abgelenkt wird, zur Bewässerung den Feldern zuführt. In den überwiegenden Fällen sind die Wasserleiten in die Erde gegraben, manche sind in den Fels gehauen, und zuweilen wird das Wasser auch durch Holzkonstruktionen geführt, die am Felsen hängen oder über Stützen laufen. Das Wallis ist an manchen Orten ausgesprochen trocken und um die mageren Felderträge zu verbessern, bot sich die künstliche Bewässerung an. Natürlich gibt es Bewässerungssysteme auch ausserhalb des Wallis, ${ }^{3}$ und die Walliser Suonen sind auch nicht die einzigen, welche Eigennamen tragen. Für Graubünden zum Beispiel sind zahlreiche Namen festgehalten in Martin Bundis junger Publikation zur Flurbewässerung (Bundi 2000: 69). In der Literatur zu den Walliser Bewässerungskanälen finden sich indessen kaum namenkundliche Erläuterungen. Man widmet sich dort zumal den Fragen nach dem Alter und den technischen Details, den Organisationsstrukturen und den rechtlichen Aspekten. Spezifische Arbeiten zur Namengebung der Suonen sind für das Wallis selten (siehe z.B. Ammann: 1995).

\section{Räumlicher Raster: Das Vorkommen künstlicher Bewässerung im Oberwallis}

In diesem Kapitel soll kurz die räumliche Verteilung der Suonen untersucht werden, darauf folgt in Kapitel 3 eine Altersbestimmung. Die Kenntnisse um geographische Verbreitung und zeitliche Tiefe werden unsere Blicke für die in der Namenlandschaft nicht immer ganz durchsichtige Situation schärfen.

\subsubsection{Orte ohne künstliche Bewässerung}

Orte ohne künstliche Bewässerungssysteme sind laut Eichenberger (1940: 5) das obere Goms bis nach Ulrichen. Bielander spricht von "wenig" Bewässerung bis Münster (1941/42: 82f. und 82 Anm. 2).

Im Vergleich zum unteren und mittleren Talabschnitt verzeichnet das obere Goms eine erhöhte Niederschlagsmenge; offensichtlich ist die künstliche Flurbewässerung hier weniger dringlich. In der Literatur gehen nun die Meinungen darüber auseinander, bis wie weit hinauf

\footnotetext{
2 Marcus Seeberger, Gymnasiallehrer in Brig und Begründer der Oberwalliser Orts- und Flurnamensammlung, hat die Lebendbelege in den Jahren 1971 bis 1973 aufgenommen; die Exzerpte historischer Orts- und Flurnamen aus Gemeinde- und Pfarrarchiven, dem Archiv des Domkapitels, dem Staatsarchiv u.s.w. legte Seeberger in den drei, vier folgenden Jahren an. Bei den Gewährsleuten im Feld ("Wieviel bezahlen Sie mir?") wie bei den Betreuern von Archiven ("Wir haben gar nichts!") begegneten dem Explorator verschiedene Schwierigkeiten; an anderen Orten waren auf Anhieb qualitativ wertvolle Aufnahmen möglich (Marcus Seeberger, 02.2002). Seit 1996 werden die Karteikarten von Martin Clausen, René Pfammatter, Valentin Abgottspon, Claude Beauge und Milda Christen sukzessive in einer elektronischen Datenbank erfasst.

${ }^{3}$ Die im folgenden angeführte Literatur enthält historische und aktuelle Belege aus Graubünden und dem Berner Oberland, Tirol und Kärnten, dem Sarganserland, dem Elsass und Savoyen. Bekannt sind in der Schweiz auch die sogenannten Wässermatten im bernischen Oberaargau; siehe Schwarze (1986: 26-37). Weiter nennt beispielsweise Martin Bundi Wasserleiten mit aufgehängten Holzkänneln für die Gemeinden Flims (Bundi 2000: 56 und 60) und Trin (2000: 61ff.). Wie Bundi ferner anhand von Irrtümern aus der jüngsten Zeit zeigt, sind wir nur schon im Alpenraum über die wirkliche Verbreitung der künstlichen Bewässerung ungenügend im Bilde (Bundi 2000: 254).
} 
im Goms die Bewässerung noch üblich sei; von den beiden oben zitierten Autoren beispielsweise nennt der eine Münster, der andere Ulrichen. Gemäss unserer aktuellen Nachfrage in den jeweiligen Gemeinden wurde in Münster noch bis in die 1950er Jahre bewässert, ebenso weiter talaufwärts in Geschinen. Auch für Obergesteln, wo es nach übereinstimmender Meinung der Literatur keine Bewässerung gegeben hat, konnten wir Lebendbelege für einstige Wasserleitungen beibringen. ${ }^{4}$

Historisch belegen lassen sich Wasserleitungen in Münster (1552 "uff der houptwasserleütten", 1575 "subtus der Houpttwasserleittenn inferiori", 1633 "uff der obren hauptwasserleiten"), Geschinen (1662 "ob der hauptwasserleiten" 1662), Ulrichen ("1618 per aqueductum vocatum Die Bachstaffollwasserleuten"5 und 1830 "ob der Wasserleiten") und Zum Loch (1669 "zum Loch by der schluocht ob der wasserleitten"). ${ }^{6}$ Für die weiter talaufwärts gelegene Gemeinde Obergesteln, bezeugen zwei Gewährspersonen das Vorhandensein von Wasserleitungen, doch konnten bislang keine historischen Belege beigebracht werden.

\subsubsection{Orte mit unregelmässiger Bewässerung}

An einigen Orten wird nicht jedes, sondern manchmal nur jedes zweite oder dritte Jahr gewässert, nämlich dann, wenn die Sommer trocken ausfallen. Eichenberger (1940: 5) nennt das Binntal, Zermatt, Saas und die oberen Regionen der südlichen Seitentäler als Beispiele. Ähnlich verhält es sich im Lötschental, wo in niederschlagsreichen Sommern wenig bewässert werden musste. ${ }^{7}$

\subsubsection{Orte mit regelmässiger Bewässerung}

In trockeneren Gegenden des Oberwallis findet sich erwartungsgemäss ein dichteres und technisch anspruchsvolleres Netz von Wasserleiten, das alljährlich von Frühjahr bis Herbst in permanentem Betrieb steht. Vor allem die rechte Seite des Rhonetals mit ihren nach Süden ausgerichteten Hängen verzeichnet westlich vom Untergoms ausgedehnte Bewässerungssys-

\footnotetext{
${ }^{4}$ Münster: Valentin Bacher (*1940) in Münster: Die Bewässerungskanäle hiessen Wasserleite f., Pl. Wasserleitige. Sie gingen vom Münstigerbach nach beiden Seiten aus. Als Knabe war er mit seinem Vater noch beim Wässern dabei. In den 1960er Jahren ist das Wässern ergangen; heute sei gar nichts mehr. Frau Werlen-Kiechler (*1935), aus Münster, heute in Geschinen, nennt Wasserleita f., Pl. Wasserleite, Dim. Wasserleitini. Geschinen: Basil Werlen (*1932) in Geschinen nennt ein halbes Dutzend Hauptwasserleiten, die einen ob dem Dorf vom Bach den Hang hinunter bis auf das Gemeindeterritorium von Münster reichend (eine über die Lokalität Wintergassen führende Leitung), die anderen oben am Berg (Wasserleitung im Trützi); mit der Mechanisierung der Landwirtschaft sei das Wässern verunmöglicht worden, die Maschinen hätten im Gelände die Leitungen zerstört Zwischen Ulrichen und Oberwald werde tatsächlich nicht bewässert, berichtet Christian Imhof, Ernen. Ulrichen: Paul Imsand (*1943) erklärt, es habe hier nie Wasserleitige (sic) gegeben, man wässere heute nicht und er hätte auch nie davon erzählen gehört, auch früher als Knabe nicht. Obergesteln: Nach anfänglich negativer Antwort erinnert sich Ida Hallenbarter (*1908), wie ihr Vater davon sprach. Er habe als Knabe noch gesehen, dass man hier bewässert habe. Maria Zeiter (*1919) erinnert sich an eine heute noch sichtbare Wasserleite am Ort Hoschtette/Gadeschtatt (auf der rechten Talseite ob dem Dorf); das Wasser sei im Milibach gefasst worden. Freundl. Mitt. Irmgard Anthenien, Brig/Obergesteln 02.2002. Oberwald: Fridolin Hischier hat weder je davon erzählen gehört noch davon gelesen; höchstens auf den Alpen könnte es das gegeben haben, doch als 12jähriger Zuhirt habe er damals bereits keine mehr gesehen.

5 Vermutlich mit Bezug zur Flur Bachstaful.

6 Zitiert nach den Karteikarten der Orts- und Flurnamensammlung Oberwallis.

7 Kippel: Gewässert hat man jeden Frühling und Sommer, und auch im Herbst für das Embd (zweiter Grasschnitt), und zwar nicht notwendig weniger, wenn es ein nasses Jahr war, denn die Suonen waren ja "im Cheer" ('im Turnus') zugeteilt und wer drankam, hat auch gewässert; man wusste ja nicht im voraus, ob es in der folgenden Zeit trocken oder nass sein werde - wir haben manchmal gewässert, während es regnete. Ignaz Bellwald, (*1940), 02.2002.
} 
teme; hier ist das Bewässern auch erwartungsgemäss am stärksten kodifiziert (geregelter Wässerturnus, schriftlich fixierte Modalitäten des Unterhalts, festgeschriebene Trägerschaft). ${ }^{8}$

\section{$3 \quad$ Zeitlicher Raster: Vom Ursprung der Suon}

Generell sind die Wasserleitungen zweifellos alt. So lässt sich etwa Homers Ilias als Beleg für ihr Vorhandensein um ca. 800 v. Chr. beiziehen:

"Wie wenn ein Mann einen Graben von dunkler Quelle zur Seite

Leitet, die Strömung des Wassers auf Felder und Gärten zu führen,

Mit einer Hacke wirft er allen Schutt aus der Rinne;

Strömt nun das Wasser voran, so schwemmt es unten die Kiesel

Alle hinweg, rasch fährt es dahin mit rieselndem Rauschen" (Homer 1920: 471).

Wann aber im Wallis erste Bewässerungssysteme angelegt wurden, ist schwer abzuschätzen. Die Autoren der Lokalliteratur nennen oft die Römer als Begründer, auch keltische Leitungen werden erwähnt, zuweilen gar solche aus dem Neolithikum. Rauchenstein zum Beispiel glaubt, aus der Semantik vom Namen der Heidenleitung in Visperterminen römischen Ursprung lesen zu dürfen (Rauchenstein 1907: 52). ${ }^{9}$ Eichenberger führt das Appellativum bisses auf kelt. bedum 'Bach' zurück, will indessen kein Datum daraus schliessen, da bedum auch als Substratwort noch zur Benennung der Wasserleitungen herangezogen worden sein könnte (Eichenberger 1940: 19). Auch die römische These wird von Eichenberger abwägend behandelt: Es würden die hohen Bevölkerungszahlen, welche man bei Julius Cäsar für das Rhonetal findet, auf eine notwendige Bewässerung zur Zeit der Römer schliessen lassen, jedoch müsse man Cäsars Zahlen mit Vorbehalt begegnen.

Auch die Naturwissenschaft versucht sich an einer Altersbestimmung der Wasserleiten. Für das Baltschieder-, das Joli- und das Gredetschtal führten die dendrochronologischen Untersuchungen zu Jahrringkurven, die 1270, 1327, 1335 und 1860 enden (Schmid 1994: 74, 86, 168, 186)..$^{10}$

Als früheste schriftlich fixierte Datierung nennt Pfarrer Seematter 1929 die Jahrzahl 930, welche an mehreren Stellen der Wyssa (die 'Weisse') in den Fels gemeisselt gewesen sei. Während der Erneuerungsarbeiten seien aber die Zeichen zerstört worden (Jossen 1989, 135f.). Analog dazu soll sich die Jahrzahl 1001 an der Restiwasserleite ${ }^{11}$ (Birgisch) gefunden haben. Belege dieser Art bleiben im Einzelnen abzuklären, dürften aber tendenziell den Anforderungen eines wissenschaftlichen Belegs nicht genügen. Quellenkritisch zu überprüfen wäre auch Bielanders Mitteilung jener chronikalisch überlieferten Nachricht von einem

\footnotetext{
$8 \mathrm{Zu}$ beachten ist aber, dass nicht jeder Wasserkanal zur Flurbewässerung dient. Es gibt auch Kanäle, die hydraulische Anlagen antreiben. Aus dem Baltschiedertal beispielsweise, wo eine zweistellige Zahl von Bewässerungskanälen gefasst und auf die Güter von Ausserberg, Baltschieder und Eggerberg geführt wurde, diente das sogenannte Dorfwasser in Baltschieder zum Antrieb von Mühlen, Walken und Sägereien und wurde daher auch Sagen-Wuor genannt; Urkunden belegen diese gewerbliche Nutzung bereits für 1517 und 1525. Auch weitere Nutzungen der Kanäle sind möglich. In Ausserberg führte die Undra (die 'Untere') Tränkwasser für das Vieh, Wässerwasser für die Wiesen und Trinkwasser für die Menschen heran. Gleichzeitig trieb dieses Wasser drei Mühlen und eine Sägerei an und diente ferner an den Waschtagen zum Waschen der Kleider; im Nachbarort St. German floss die Niwa auf zwei Mühlen, eine Sägerei und das 1917/18 erstellte Elektrizitätswerk.

9 Schwzdt. Heid 1. 'Angehöriger einer vorchristlichen Bevölkerung oder nichtchristlichen Gruppe', als Bestimmungswort von komponierten FlN "meist Örtlichkeiten bezeichnend, an welche sich Sagen von einer untergegangenen Bevölkerung knüpfen, wie auch Funde von Altertümern seither den Volksglauben vielfach bestätigt haben"; 2. 'Flur mit oder ohne Heidekraut' (Id. 2: 985ff).

${ }^{10}$ Da bei diesen Proben die Waldkante fehlt, und somit das Fälldatum nicht genau eruiert werden kann, ist es möglich, dass diese Hölzer einige Jahrzehnte nach dem genannten Datum gefällt und in die Suonen eingebaut wurden. Freundl. Mitt. Prof. Dr. Hanspeter Holzhauser, Zürich.

${ }^{11}$ Zum Namen siehe 5. 2. d.
} 
Wasserleitungsstreit im Goms aus dem Jahr 1008 (Bielander 1941/42, 83) sowie die Nachricht Schmids von einer Urkunde aus dem Jahr 1040, welche die Verteilung des Augstbordwassers (wird am Augstbordbach in der Gemeinde Embd gefasst) geregelt hätte (Schmid 1994: 157 u. 11).

Stichhaltig sind die Urkunden aus dem 13. Jh. mit Erwähnung der Wasserleitungen. Hans Robert Ammann weist indessen zu Recht darauf hin, dass diese Urkunden zur Datierung der Wasserleitungen nicht herangezogen werden dürfen, weil im Ganzen für das Wallis nur sehr wenige schriftliche Zeugnisse vorliegen, die älter sind (Ammann 1995: 262). Das Erscheinen der Wasserleiten in den schriftlichen Zeugnissen des 13. Jh. dürfte also eher von der Verdichtung der schriftlichen Kultur als von der Begründung eines Bewässerungssystems abhängen. In den ältesten vorhandenen schriftlichen Belegen lauten die Appellativa für Wasserleitungen auf lat. aqua ('Wasser'), aqueductus ('Wasserleitung') oder canalis ('Röhre, Rinne, Kanal').

1245 "...vendidi...vineam...et dimidiam grangiam cum casali circumadiacente et aqua ad irrigandum supradicta..."

1252 "...vendidi pratum meum...et aquam eidem prato pertinentem..."

1255 "...que terra est annexa vinee...et protenditur usque ad aqueductum directo tramite..."

1261 "...unum sectorium prati supra canalem..."

1278 "...aque ductus per pratum domini apud Morgiam..." (Eichenberger 1940: 20f.)

Ammann fand in Privaturkunden 15 weitere Belege für die Zeit zwischen 1245-1300. Für die Zeit zwischen 1301-1400 bringt Ammann 140 Belege.

Die ersten Eigennamen von Suonen werden im endenden 14. Jh. greifbar. Hier tauchen auch die ersten Erwähnungen der Appellativa Suon und Wasserleite auf.

1385 "aquam in aqueductu qui vocatur kuntzwasserleita" (kuntz: PN) (Brigerbad)

1388 "aquam in aqueductu ... in die veneris . qui vocatur huopsun" (Das Bestimmungswort bezieht sich vermutlich auf einen FlN) ${ }^{12}$ (Täsch)

1390 "in aqueductu cui dicitur superior brigarra" (in den übrigen Belegen: Brigeri 'Brigerin') (Brig)

1393 "octavam partem aque...in aqueductu que vocatur gornarra" (heute: die Gorneri 'die Gornerin'; vermutlich in Bezug zum historischen FIN Gorner) ${ }^{13}$ (Filet)

Man darf in der Frage nach dem Ursprung der Walliser Wasserleiten wohl mit Denis Reynard übereinstimmen: "C'est une question qui reste d'ailleurs ouverte, malgré les tentatives d'explication plus ou moins heureuses de nombreux érudits." (Reynard 2002: 31). Nachzuweisen ist dagegen eine entschiedene Verdichtung des Bewässerungssystems im 15. Jh., die abhängt von einem entsprechenden Ausbau der landwirtschaftlichen Nutzung während dieser Zeit (Reynard 2002: ibid).

\section{Die zwei verbreitetsten Appellativa: Suon und Wasserleite}

Es scheint, die unterschiedlichen Appellativa Suon und Wasserleite benennen keine spezifischen Konstruktionsmerkmale der Kanäle (z.B. im Boden verlaufend oder in Holzkänneln gefasst). Ein Rückschluss von einem der zwei Appellativa auf die Konstruktionsweise der Leitung lässt sich also nicht herstellen.

\footnotetext{
12 Mhd. huobe f. 'Stück Land von einem gewissen Masse'.

13 Aus den Dateien der Orts- und Flurnamensammlung Oberwallis.
} 


\subsection{Suon f.}

\subsubsection{Lebendbelege}

In der umfangreichen Literatur erscheint meist schriftsprachlich Suon mit dem Pl. Suonen. Schwzdt. gibt es zahlreiche Varianten: Süon, Suen, Süan, Sua, Sü̈, Süe; das Idiotikon (7: 1109) nennt weiter die Diminutivformen Süenli (Wallis) und Sü̈̈lli (Piemont: Alagna), und der Schweizerdeutsche Sprachatlas bringt für die walserischen Südorte Sieli (SDS III: 155, Spalte 4). Für das Lötschental (Ferden, Kippel und Wiler) erfuhren wir die Variante Siänd$\operatorname{li}(n)$ als Lebendbeleg. ${ }^{14}$

Das Vorkommen von Suon in Brig, Visp und den Vispertälern sowie in Leuk, wie es in der Literatur angeführt wird, ${ }^{15}$ widerspricht unserem Wissen über die Region. In diesen Gebieten ist zumindest heute stets nur Wasserleite gebräuchlich. Dieser Widerspruch veranlasste unsere Umfrage. ${ }^{16}$ Die Resultate aus den einzelnen Gemeinden lauten:

\section{Westlich Raron}

Ausserberg: Pl. Siene (Felix Schmid 1981: 30), ebenfalls bei Maurus Schmid (1994: 45). Bei der mündlichen Umfrage (02.2002) verwendet Raphael Schmid (*1925) spontan den Begriff Wasserleita, Pl. Wasserleite, sagt aber später, Wasserleite habe man vor allem geschrieben, und Süe, Pl. Süene (sic) habe man gesprochen. Maurus Schmid (1994: 126) schreibt, dass eine der ältesten Suonen von Ausserberg "früher einfach $d$ Wasserleita" geheissen habe und an ihr heute die Undra ('die Untere') und auch der Weiler Wasserleita liege.

Steg: Süenu f., Pl. Suenä, nie Wasserleite (Lina Zengaffinen, *1921, 02.2002). Am Berg sage man Süe, Pl. Süene; im Grund würden die grossen Kanäle Rü̈̈ss heissen, die mittleren Wasserleitu und die ganz kleinen Schrapf (Simon Kalbermatter, *1932, 03.2002.)

Niedergesteln: Süe, Plural Süene (Dario Steiner, *1949, 10.2001). Maurus Schmid (1994: 70) erwähnt eine Sandwasserleita, die am Ausgang der Jolischlucht gefasst wird und in die Ebene westlich unter dem Dorf führt. Hierbei handelt es sich gemäss Auskunft von Dario Steiner um die einzige Süe in der Gemeinde, die den Namen -wasserleite trägt.

Ferden: Suän, Pl. Siänä, Erwin Werlen, *1913; äs Siändlin, Rosa Werlen-Bloetzer, *1934.

Kippel: Suän, Pl. Siänä, Dim. Siändli. "Wasserleite sagte man im Dialekt nie, aber in den Schriften habe ich den Ausdruck schon gesehen" (Ignaz Bellwald, *1940).

Blatten: Suän, Pl. Suänä, "Wasserleite hörten wir nie" (Karl und Maria Bellwald-Siegen, *1926 und 1929).

Raron: Wasserleita., Pl. Wasserleitä seien die Feinverteiler auf dem Grundstück; ä Süe., Pl. Süenä dagegen seien die grossen Hauptleitungen, die "dr Schwall Wassr" (die grosse Menge Wasser) bringen. Man kenne in der Gemeinde beide Begriffe und unterteile so nach der Grösse des Kanals (Christof Salzgeber). Bestätigt durch Werner Imboden, *1926, 03/2002: Wasserleita, Pl. Wasserleitä seien im Grund, oben am Berg sage man Süe, Pl. Siene. (Imboden zeigt während des Gesprächs über die Siene auf die Kanäle, die das Wasser aus der Felsschlucht des Bitschi herausführen.)

Bürchen: Wasserleita f., Pl. Wasserleite.; ein grösseres Werk mit mehr Wasserführung heisse Wasserleitig; von Suon sei in Bürchen nie die Rede, ausser im Falle des Ginalswasser, dem man auch "d alti Süe" sage (Oswald Zenhäusern, *1932, heute wohnhaft in Visp). Dies entspricht einem älteren Literaturbeleg, wonach die Ginanzerin, aus dem Ginanztal, auch "die alte Suon" genannt wird (Stebler 1922: 74).

\footnotetext{
${ }^{14}$ Freundl. Mitt. Ignaz Bellwald (*1940), Kippel 03/2002.

15 Laut Paul Zinsli (1976: 188) "ist das Wort (Suon) für die Wasserzuleitung nur von der Sprachgrenze an bis Visp bekannt, ferner in Lötschen und in den Vispertälern." Das Idiotikon (7: 1109) will Suon auch für die Bezirke Brig, Visp und Leuk belegen, lehnt es aber für das Goms richtigerweise ab. Auch gemäss Bielander (1941: 42, 79, Anm.3) fehlt der Ausdruck Suon im Goms.

16 Befragt wurden Angehörige einer älteren Generation; bei jüngeren InformantInnen handelt es sich um solche, die im kulturellen Bereich engagiert und mit den örtlichen Verhältnissen vertraut sind. Die (mehrheitlich telefonische) Umfrage wurde zwischen November 2001 und Februar 2002 durchgeführt und bestand aus einem Gespräch mit fünf, sechs offenen Fragen zu Terminologie und Verwendung der Bewässerungskanäle.
} 
Unterbäch: Mit Suon bezeichne man die grossen Kanäle, die in den Bächen angeschlagen werden, und Wasserleiten hiessen die kleineren Kanäle, die zu den Wiesen führen (Gregor Zenhäusern, 01.2002). Ebenfalls für Unterbäch nennt Id. 7, 1109 Pl. Siene.

Es scheint, Suon sei in einem begrenzten Gebiet des westlichen Deutschwallis verbreitet, das in etwa den Bezirk Westlich Raron beschreibt. Nur in einer Handvoll von Gemeinden sei ausschliesslich der Begriff Suon üblich; dieses Kerngebiet konzentriert sich, soweit das anhand der limitierten Umfrage abgeschätzt werden kann, auf den Raum Niedergesteln. Daneben gäbe es Übergangsgebiete wie die Gemeinde Raron oder die Gemeinden der Rarner Schattenberge, wo beide Begriffe geläufig sind.

\subsubsection{Historische Belege}

Für das ausgehende 14. und für das 15. Jh. enthalten die Dateien der Orts- und Flurnamensammlung Oberwallis je zwei Belege für komponierte Wasserleitungsnamen, die als Grundwort das Appellativum Suon enthalten. Im 16. Jahrhundert kommen sieben andere dazu. ${ }^{17}$

1388 in Täsch "aquam in aqueductu ... in die veneris qui vocatur huopsun"18

1396 in Unterbäch "tangit terram cui dicitur Wildinsuon" (Bestimmungswort vermutlich in Bezug zum historischen FlN "wyldin")

1449 in Bürchen "sub aqueductu cui dicitur alt suen"

1495 in Eischoll "In aqueductu eorum de oysel [=Eischoll] cui dicitur die niw suon" (mhd. niw, niuw, nuw 'neu')

1513 in Blatten/Lötschen "die nuwen suon ... inferius die brunne suon" (vgl. den historischen FlN "an brunnmatten"19) (1646 in Blatten)

1544 in Unterbäch "inter aqueductus quibus dicitur finilsuon (Bestimmungswort vermutlich in Bezug zum historischen FIN "in den finillun"20)

1545 in Unterbäch "die nüwen suen" und in Bürchen "die byelsuon" (Bestimmungswort vermutlich mit Bezug auf den FIN Biel)

1556 in Unterbäch "vocata die alten suon"

1570 in Eischoll "aquaeductum vocatum die altt suonn"

1572 in Eischoll "ad aqueductum vocatum mutter suon" (Vermutlich in Bezug zum historischen FIN "Mutter acher")

1578 in Bürchen "die Byffig suon" (Bestimmungswort vermutlich mit Bezug zum FlN Bifig m.)

Bei diesen frühen Belegen fällt die Konzentration auf die drei Orte Bürchen, Unterbäch und Eischoll auf; nur je eine Nennung betrifft das Lötschental (Blatten) und das Nikolaital (Täsch). Für das 17. Jahrhundert sind einige Dutzend weitere Belege vorhanden; auch sie und die Belege des 18. und 19. Jahrhunderts konzentrieren sich auf die drei Gemeinden in den Rarner Schattenbergen, das Lötschental und Niedergesteln.

\subsubsection{Wortverbreitung und Wortbedeutung}

Suon oder Suen ist laut Zinsli (1976: 187f.) "ein im westschweizerdeutschen Bergmundartbereich bekannter Ausdruck für künstlich erstellte Bewässerungsgräben", wie er sich in historischen Belegen auch 1503 im St. Galler Oberland, 1667 im Elsass, 1425 (und in weiteren Jahren) im Berner Oberland findet. Glatthard (1987: 3f.) führt Beispiele aus dem Wallis,

\footnotetext{
17 Detaillierte Angaben (Archiv, Urkundenstück mit Signatur, Ausstellungsort des Dokumentes usw.) zu den jeweiligen historischen Belegen finden sich im Zettelkatalog sowie neu in der elektronischen Datenbank der Orts- und Flurnamensammlung Oberwallis in Brig.

18 Das Bestimmungswort bezieht sich vermutlich auf einen Flurnamen. Mhd. huobe f. 'Stück Land von einem gewissen Masse'.

${ }^{19}$ Mhd. brunne m. 'Quell, Quellwasser, Brunnen'.

${ }^{20}$ Vgl. schwzdt. Finel, bern. Finiz, rom. foenils 'Heuschuppen, Heuscheuer(n) (ONB 2, 142/VRom III: 79).
} 
Piemont, Elsass, Schwaben, Tirol und Kärnten an. Die appellativische Verwendung indessen beschränkt sich heute auf den alemannischen und südbayerischen Raum (Glatthard 1987: ibid.).

Für das Wallis konzentrieren sich die historisch belegten Suon-Bezeichnungen auf den Bezirk Westlich Raron, auf einen kurzen Talabschnitt etwa vom Raum Steg bis unterhalb Visp; die Resultate unserer Feldforschung weisen auf einige Gemeinden im Umkreis von Raron. In FIN taucht der Begriff sehr selten auf: So als Bestimmungswort eines Kompositums in Suonacher ('Suonacker', siehe 6.8) und als Substantiv im Dat. Pl. mit vorangestellter Präposition zi Siänun ('bei den Suonen'; d. i. in Ferden der Ort, wo die Ferdenriedsuon und die Oberdorfsuon angeschlagen werden). ${ }^{21}$ Die Verbreitung von Suon im Wallis scheint also de facto recht gering zu sein.

\subsubsection{Etymologie}

Das Schwäbische Wörterbuch erklärt die Etymologie von Suon als "ungeklärt" (Schwäb. Wb. 5: 1592). Das Idiotikon nennt es ein "ungermanisches Wort" und tritt nicht weiter auf die Etymologie ein (Id. 7: 1109). J. U. Hubschmied bringt eine Herleitung aus dem Gallischen (Rübel 1950: 87). Er konstruiert ein gall. *Suagnis f. 'schöne Leitung = künstliche Leitung' aus dem Stamm -ag (lat. ag-ere, griech. ag-ein, anord. $a k-a$ ) und den Präfixen gall. *ande und *su. *Su-agnis erscheine im Ahd. als Lehnwort *suon(a). Da Hubschmied auf unbelegte Worte zurückgeht, die er aus einem Stamm und Präfixen konstruiert, ist seine These nur schwer überprüfbar. Rübel übernimmt sie ohne Vorbehalt (Rübel 1550: ibid.), Egli begegnet ihr mit einigem Zweifel (Egli 1984: 128). Populär geworden ist eine andere Etymologie, nämlich die Rückleitung von Suon auf ahd. suona 'Sühne, Urteil, Gericht'. Fragwürdig ist hier nebst der daraus zu schliessenden Apokope ${ }^{22}$ der semantische Zusammenhang. Er wird von Sigismund Furrer damit erklärt, dass die Gemeindeversammlungen, bei welchen ursprünglich auch Gericht gehalten wurde, an Orten organisiert worden seien, wo man ohnehin zusammenkam, also etwa bei den gemeinsamen Wasserleitungen. Das ahd. Appellativum für das Gericht wäre sodann auf den Ort des Gerichts übergegangen (Furrer 1850: 94). In der Heimatkundeliteratur und in der Presse ist Furrers Etymologie weitergesponnen worden zu den Berichten, wonach Strafgefangene die gefährlichen Strecken der Suonen gebaut hätten (Wendt 2001). Glatthard (1987: 3ff.) will unser Wort von ahd. suoha f. 'Egge, Furche' herleiten. Und in der Tat erscheint im Tirol mda. Sueche f. als Appellativum für die 'Wasserleitung auf dem Felde' (Schöpf im Tirol; Id. 1866, 728). Für das ahd. suoha f. sei zudem eine Diminutivform suoli belegt, die Schwund des intervokalischen Reibevelars und anschliessende Silbenkontraktion bezeuge. Konstruiert man denselben Konsonantenschwund mit Silbenkontraktion für die unabgeleitete Form, so erhält man Suo, welches bei Zinsli belegt ist als Südwalser Appellativ (Zinsli 1976: 187f.). Belegte Nom. Pl.-Formen von suoha lauten suohun (nDeklination), woraus Glatthard die entsprechende Form mit Schwund des Inlautkonsonanten konstruiert: *suon. Die weitere Entwicklung erklärt er sich durch Analogiebildung: Geht man von der obgedachten Prämisse des Nom. Pl. *suon aus, erhält man eine Isolation des Nom. Sg. suo unter den übrigen Deklinationsformen, die nunmehr, mit Ausnahme des Gen. Pl. *suono, allesamt gleich auf *suon lauten. Unter diesem Druck würde sich der Nom. Sg. der Form *suon anpassen. Da aber in diesem Fall die Numerusopposition entfiele, würde später eine weitere Anpassung erfolgen, diesmal an die o-Deklination, die im Schwzdt. eine Numerusopposition aufweist. So erhält man: Nom. Sg. Suon - Nom. Pl. Suone. Beide Analogiebildungen sind für andere Worte belegt. Freilich könnte man den n-Auslaut auch einfacher

\footnotetext{
21 Interview mit Erwin Werlen (*1913) in Ferden, 04.2002.

${ }^{22}$ Es ist mhd. zu suone f. ein gleichbedeutendes Wort suon m. belegt, das aber wohl nicht durch Apokope aus suona sondern durch eine im Vergleich zu suona abweichende Wortbildung entstanden ist. Für unsere Etymologie dürfte das Wort nicht in Betracht kommen, schon der Semantik wegen, aber auch weil es mask. Genus hat.
} 
erklären, als dies Glatthard tut, nämlich als reguläre Form des lokativischen Dativs. In diesem Fall hätte sich ein Flurname, z. B. 'an der Wasserleitung', zur nominativen Bezeichnung der Wasserleitung selbst entwickelt. Diskussionswürdig bleibt in Glatthards Etymologie die Annahme von einem Schwund des velaren Frikativs. Eine künftige Etymologie des Worts müsste vielleicht auch das vorröm. engad. Suona 'Kübel' in Betracht ziehen (REW: 7578).

\subsection{Wasserleite f.}

\subsubsection{Lebendbelege}

\section{Leuk und Umgebung}

Agarn: Wasserleitu f., Pl. Wasserleitä (Susanne Hugo-Lötscher, *1960).

Leuk: Wasserleitu f., Pl. Wasserleitä; nie Suon (Irma Andenmatten-Villa, *1926).

Erschmatt: Wasserleitu, unsicher: Pl. Wasserleitige, nicht Suon (Edmund Steiner, *1956). "Suon - das sagen wir nicht, das ist welsch." (Gregor Schnyder, *1922).

Turtmann: Wasserleitu, Pl. Wasserleitä, nie Suon (René Meyer, *1952, aufgewachsen in Turtmann).

Oberems: Wasserleitu, Pl. Wasserleitä; "Suon ist uns nicht bekannt" (Raphael Biffiger, *1946, Sitten; aufgewachsen in Oberems).

Gampel: Wasserleitu, Pl. Wasserleitä; "Suon haben wir nicht gebraucht" (Kurt Schnyder, Niedergampel, aufgewachsen in Gampel). Simon Kalbermatter aus Steg (*1932) berichtet, dass er 1953-61 in Gampel gearbeitet habe und einmal in einem Gespräch feststellte, wie das Wort Suon hier gar nicht bekannt war. "Ä Süe, was de das si?" ('eine Suon, was denn das sei?'), fragten ihn die Gesprächspartner aus Gampel, die sowohl für die Bewässerungskanäle am Berg wie auch im Grund ausschliesslich Wasserleitu sagten.

\section{Visp, Umgebung Visp und Vispertäler}

Grächen: Wasserleita, Pl. Wasserleite; Suon heisse es in Grächen nie (Reinhard Walter, *1939, 11.2001).

Visperterminen: "Es hiess immer Wasserleita, Suon ist für mich ein Fremdwort" (Gerold Vomsattel, *1964, 02.2002).

Saas-Fee: $\ddot{a}$ Wasserleita, Pl. $d$ Wasserleite (Werner Imseng, *1924, 12.2001).

Visp: "Wir sagen Wasserleita, Wasserleite/ä und die von Törbel sagen Suonen, für Zeneggen weiss ich es nicht" (Maria Antonietti-Wyer, *1923).

Eyholz: "Wir sagten Wasserleita und Pl. Wasserleite, Suon war uns ein Fremdwort." (Louis Albrecht, Visp, *1932, aufgewachsen in Eyholz).

Eggerberg: In der Arbeit von Karl In-Albon (In-Albon 1997) ist immer nur von Wasserleita, allenfalls von Wasserfüor die Rede, auch das bei ihm zitierte historische Dokument, die Dorfwasserschrift von 1678, spricht von Wasserleiten (S. 26), der Begriff Suon kommt nie vor.

\section{Brig und Umgebung}

Simplon-Dorf: Immer Wasserleita, Pl. Wasserleitä; Suon gebe es nie (Erich Jordan, 01.2002).

Naters: Wasserleita, Pl. Wasserleitä, Suon sage man nicht (Ignaz Mutter, *1929).

\section{Goms}

Ernen und Grengiols: laut mündl. Auskunft von Christian Imhof 12.2001 und einer Informantin aus Grengiols immer nur Wasserleiten, nie Suonen.

Obergesteln: Wasserleitä/e (Irmgard Anthenien, 02.2002).

Für das gesamte Obergoms siehe die Belege in Anm. 4.

\subsubsection{Historische Belege}

Prominentester historischer Beleg ist eine Passage in der 1572 verfassten Autobiographie von Thomas Platter (ca. 1499-1582) aus Grächen. In seinen Kindheitserinnerungen schreibt er von "einer wasserleitten, do man das wasser den bergen nach zue den güettren füert" (Platter 1999: 31). Bis ins 14. Jahrhundert reichen mehrere Belege in den Dateien der Orts- und 
Flurnamensammlung Oberwallis, z.B. "in aqueductu qui dicitur gorpwasserleita" (Bestimmungswort vermutlich in Bezug zum FIN Gorp) Naters 1390 oder "aqueductu cui dicitur du heydenschu (ahd. heidanisk 'heidnisch', mhd. heidenisch 'heidnisch' schwzdt. heid(n)isch 'Überreste vorchristlicher Bauten bezeichnend, heidnisch, alt'; Id. 2: 987), "wasserleyta de Nantz" Visperterminen 1305. Die Schreibweisen variieren zwischen

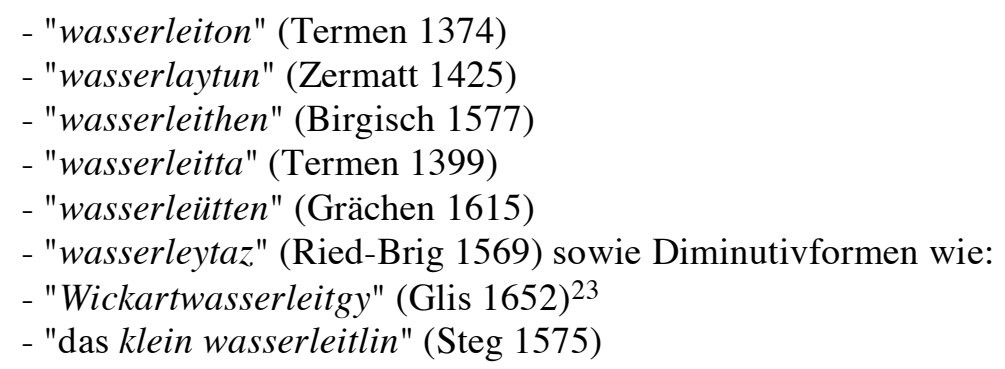

\subsubsection{Wortverbreitung und Wortbedeutung}

Die geographische Verteilung der über 400 historischen Wasserleiten-Belege aus den Dateien der Orts- und Flurnamensammlung Oberwallis (Zeitspanne: 14. bis beginnendes 20. Jh.) zeigt eine Konzentration

- im Raum Brig mit 128 Belegen (Brig, Glis, Naters, Mund, Birgisch, Brigerbad, Ried-Brig, Termen und Simplon),

- im Raum Visp mit 92 Belegen (Visp, Stalden, Törbel, Zeneggen, Visperterminen, Eyholz, Baltschieder, Eggerberg, Lalden) sowie

- im Untergoms mit 69 Belegen (Ernen, Binn und Ausserbinn, Martisberg und Lax, Fiesch und Fieschertal, Bellwald, Niederwald, Mühlebach, Steinhaus) und

- im Gebiet um Mörel mit 43 Belegen (Mörel, Ried-Mörel, Bitsch, Filet, Betten, Bister, Grengiols).

Gegenüber der intensiven Verbreitung im Raum Visp-Brig-Untergoms zeichnen sich Gebiete $\mathrm{ab}$, in denen das Apellativum Wasserleite signifikant schwächer belegt ist:

- die Gegend um Leuk zählt 35 Belege (Leuk, Agarn, Oberems, Ergisch, Guttet und Feschel, Bratsch, Erschmatt, Albinen, Varen, Salgesch),

- das Nikolaital 31 (Zermatt, Täsch, Randa, St. Niklaus, Grächen, Embd)

- das Obergoms verzeichnet nur 25 Angaben (Blitzingen, Selkingen, Biel und Ritzingen, Gluringen, Reckingen, Münster, Geschinen und Ulrichen), was auf die geringe bis fehlende Bewässerung zurückzuführen ist.

- die Gegend um Raron zählt nur 21 Belege (Raron, Steg, Ausserberg, Niedergesteln, Lötschental, Bürchen, Unterbäch), da vermutlich die Beharrungskraft des älteren Begriffs Suon die stärkere Ausbreitung von Wasserleite verhindert hat.

Es muss bei diesen historischen Belegen die Möglichkeit eingeräumt werden, dass bei der Wortwahl gelegentlich die Herkunft der Kanzlisten ausschlaggebender gewesen sein könnte als die am Ort tatsächlich für die Bewässerungskanäle gebräuchliche Bezeichnung. Eine Bestätigung dieser Vermutung findet sich z.B. in der Gemeinde Ferden: Hier wird 1665 "Zur

\footnotetext{
23 Von PN Wighari (Förstemann 1, 1584) stammt das Bestimmungswort vermutlich nicht ab, obgleich unsere vier ältesten Belege lauten "wicarbuel" (Glis 1279) "wikarsaker" (Naters 1320), "wikars" (Naters 1320) und wikarsacher (Brig 1374), denn den a-Vokal würde man in einem solchen Fall umgelautet erwarten, er bleibt aber in unserem Fall bis ins 18. Jh. fest. Man müsste von einem erstarrten PN ausgehen, um diese These zu erhalten. Genitives s fehlt in den meisten Belegen. Das auslautende t, das ab 1374 in unseren Quellen erscheint (wikart, Brig 1374), könnte als gängige epithetische Bildung gedeutet werden (Mittelhochdeutsche Grammatik, § 149). Vielleicht sollte man eine Form mit Suffix ahd. -ari vermuten, z. B. als Nomen agentis mit einem mhd. wicke 'schlimme Künste, Zauber'. Nicht in Frage kommt eine Herleitung von spmhd. vicar 'Vikar', denn dieses Wort wird erst im 14. Jh. aus dem Lat. entlehnt (Kluge 1995, 864) und weist bis heute Zweitsilbenbetonung auf.
} 
wasserleuten" (im Oberwallis häufige Schreibweise für Wasserleite) fassbar, und noch 1884 heisst es im Nachbardorf Kippel "die Ferdanwasserleite". Im örtlichen Dialekt aber ist heute immer nur von der "Färdasuän" die Rede, und Wasserleite war allen Gewährsleuten ein unbekannter Begriff. ${ }^{24}$ Auch ist im Pfarrarchiv Kippel 1548 die "ferdensuon" schriftlich belegt. ${ }^{25}$ Als 1587 in Anwesenheit mehrerer Talleute (die Geschlechter stammen ausschliesslich aus den Lötschentaler Gemeinden) im Haus von Fähnrich Meyer in Kippel ein Vertrag aufgesetzt wird, ${ }^{26}$ werden Suon und Wasserleite synonym verwendet - der Notar jedoch heisst Nikolaus Perren und stammt aus Turtmann, sprachgeographisch (heute) ausschliesslich im Wasserleitengebiet. Denkbar ist, dass die historischen Wasserleitenbelege in den Suonenlandschaften auf diese Weise entstanden bzw. vereinzelt auch in die Umgangssprache Eingang fanden. ${ }^{27}$

Trotz solcher Vorbehalte vermögen aber unsere historischen und mündlichen Belege die geographische Verteilung tendenziell anzuzeigen.

\subsubsection{Etymologie}

Im östlichen Oberwallis sagt man Wasserleita. Dies entspricht im Grundwort der ahd. Form leita 'Leitung', sachbezeichnendes Femininabstraktum zum ahd. Verb leitan 'leiten, führen'. Das Wort ist etymologisch durchsichtig und auch seine Anwendung zur appellativischen Bezeichnung von Bewässerungskanälen ist klar.

Elisa Wipf bringt für Visperterminen die Variante Wasserleiti zu ahd. leiti 'Leitung, Führung', einer im Vergleich zu leita unterschiedlich gebildeten Substantivierung aus demselben Verb ahd. leitan.

\subsection{Suon und Wasserleite: Die sprachgeographische Grenze und ihre Bewegung}

Gelegentlich wird in der Literatur pauschal auf das Vorkommen zweier verschiedener Ausdrücke - Suon und Wasserleite - im Oberwallis verwiesen. Wo geographische Zuweisungen gemacht werden, handelt es sich meist um grossräumige und mitunter fehlerhafte Angaben. Die Aussagen innerhalb der Literatur sind gegensätzlich, und unsere Felddaten bringen nochmals andere Ergebnisse. Diesen gemäss beschränkt sich der Begriff Suon aktuell auf den zweituntersten deutschsprachigen Bezirk (Westlich Raron) (vgl. 4.1). Unterhalb Raron ist im Bezirk Leuk und oberhalb Raron etwa von Visp an der Begriff Wasserleite gebräuchlich. Die östliche Übergangszone von Suon zu Wasserleite würde also in etwa mit der Lautgrenze ee/ää (z.B. schweer-schwäär) zusammenfallen, die ebenfalls im Raum Visp liegt. ${ }^{28}$

Im Fall des Appellativums Suon werden die Ergebnisse unserer Feldforschung von historischen Belegen bestätigt. Mit Ausnahme der 1388 für Täsch belegten "huobsuon" situieren sich alle Suonbelege in den Rarner Schattenbergen, im Lötschental und in Niedergesteln (siehe die Auflistung unter 4.1). Umgekehrt ignoriert aber Wasserleite die von uns supponierte Suon-Wasserleite-Grenze im Raum Visp und erstreckt sich talabwärts durch die ganze

\footnotetext{
24 Ignaz Bellwald, *1940, Kippel: "Es hiess immer die Färdäsuän, niemand hörte ich Färdäwasserleita sagen."

25 Pfarrarchiv Kippel, Hertingerjahrzeit, 1548: S. 56.

26 Burgerarchiv Kippel, Signatur E3, Kopie des Vertragswerks vom 3. Juli 1587.

27 Weitere Beispiele von schriftlichen Wasserleitenbelegen aus dem Lötschental lauten: In Kippel am Ort Haispiel das Grundstück von Johann Kaiser namens "krummwasserleite Gut" (Gemeindearchiv Kippel, altes Registerbuch 19.Jh.); heute spricht man am Ort von der Chrummsuän. In Blatten am Ort Zum Hals die "wasserleitung die neiwe sun" (Pfarrarchiv Kippel, Anniversarium des Nikolausaltars in Kippel, 7. Januar 1616). (Freundl. Hinweise Ignaz Bellwald, Kippel).

28 Vgl. die Karten im SDS 1: 73ff.; auch: Zinsli (1976: 176f.). Ebenfalls die Karten 4 und 5 im Anhang von Rübel (1950).
} 
Suonenlandschaft. ${ }^{29}$ Damit stehen die historischen Belege für den Fall des Appellativums Wasserleitung im Widerspruch zur heutigen Sprachlandschaft. Wie bereits erläutert, kann dies mit dem Wortgebrauch der Kanzlisten zusammenhängen. Mit dem Übergang von der lateinischen zur deutschen Kanzleisprache wird zunehmend das pauschale Appellativum Wasserleite für lat. aquaeductum verwendet. Eine hieran sich schliessende These wäre, dass Wasserleite erst von der Kanzleisprache aus in den Dialekt drang.

Glatthard (1987: 9) erklärt das Zurückweichen von Suon als "Regression im höchstalemannischen Raum", bei der im Berner Oberland wie im Goms der Worttypus Suon verloren gegangen und im Goms durch Wasserleite ersetzt worden sei. Das altalemannische Suon sei dabei als frühmittelalterliches Relikt erhalten geblieben. In der Region Raron ist es noch heute lebendig. ${ }^{30}$

In der Gegenwart bewegt sich die Sprachlandschaft freilich auf ganz andere Art. Zunehmend tauchen unetymologische Benennungen auf. In Saas-Grund etwa bietet der örtliche Verkehrsverein heute seinen Gästen "Wandern an heiligen Wassern" an, durch den Furrwald bis zur Triftalp entlang einer Suone (sic!). ${ }^{31}$ Hier war früher nur die Bezeichnung Wasserleite gebräuchlich. ${ }^{32}$ Auch im industriellen Umfeld lassen sich solche Wechsel der Appellativa beobachten. In Steg/Hohtenn, wo für Bewässerungskanäle an Berghängen ausschliesslich von Suonen die Rede ist, wird ein Kanal ds Wasserleitji genannt. Es zweigt von der Druckleitung des Lonza-Kraftwerks ab, da wo früher die alt Süe am Ort Chloschterli aus der Lonza gefasst wurde. Der Namenwechsel erfolgte also mit dem Bau der neuen Leitung, die nicht mehr am Bach gefasst wurde, sondern an der Druckleitung der Lonza-Fabrik. ${ }^{33}$ Weil viele der jüngeren

29 Z. B. in Raron 1653 gruebwasserleita; Ausserberg 1377 wingartwasserleita (ahd. wingarto, mhd. wingart, Kompositum aus win 'Wein' und gart 'Garten' = 'Weinberg'); Niedergesteln 1393 spitzboden wasserleita; Steg 1396 talwasserleita; Kippel 1884 Ferdanwasserleite; Ferden 1665 zur wasserleuten.

30 In Täsch wird die Bezeichnung Wasserleite gemeinsam mit dem erwähnten Beleg der "huobsuon" 1388 aktenkundig: 1388 hupowasserleite und huopwasserleite,1389 hubwasserleite - alle vier Belege beziehen sich auf den selben Bewässerungskanal. Könnte es sich hier um einen Beleg für das Vordringen des Begriffs Wasserleite in das damals noch grössere Verbreitungsgebiet von Suon handeln?

Sprachgeographisch eindeutig im Suonengebiet liegt Niedergesteln. Die Bewässerungskanäle heissen hier Süe, Pl. Süene, doch ein (heute verlassener) Weiler am Hang östlich ob dem Dorf trägt den Namen Wasserleite und einem Niedergestler sagte man $d r$ Wasserleitu-Hans (freundl. Mitt. Gabriel Imboden, 12.2001). Ist hier Wasserleite bereits in das letzte Walliser Rückzugsgebiet von Suon vorgedrungen?

31 Gastinfo Ferienort Saas-Grund 2001: 5.

32 Umgekehrt kann der Tourismus in anderen Fällen Appellativa oder Namen bewahren, die sonst der Vergessenheit anheim fallen würden. In Grächen etwa traversieren vier übereinanderliegend angeordnete Wasserleiten (die Bineri, die Driijeri, die Chilcheri und die Eggeri) die ganze Bergflanke vom Talkessel des Riedgletschers bis zum Dorf und reichen teils bis zum nördlichen Ende des besiedelten Plateaus (die oberste Wasserleite namens Eggeri). Ihre Länge variiert zwischen drei und sechs Kilometern. Die Einheimischen begehen die Waldwege an den Leitungen seit jeher, um zu ihren Alpen, Weiden und Holzsschlägen zu gelangen. Heute sind sie eine der Hauptattraktionen für Sommergäste, und auf Holzschildern lassen sich die jeweiligen Namen der Wasserleitungen ablesen. Wer indessen am Grächerberg noch wässert, tut dies mit grossen Spritzvorrichtungen, die allenfalls in den Wasserleiten gefasst werden. (Wo der Druck des Gefälles von der Wasserleite auf die darunterliegenden Sprinkleranlagen nicht ausreicht, werden Pumpen installiert). Dagegen sind im Lötschental in den letzten zwei, drei Jahrzehnten fast alle Suonen verwachsen und durch Fahrrinnen der landwirtschaftlichen Fahrzeuge unterbrochen. Hier war die Notwendigkeit des Bewässerns nie zwingend wie in den niederschlagsarmen Vispertälern, und heute wässert selbst in trockenen Sommern kaum mehr jemand, und wenn, dann mit Pumpen und Zerstäubern, die das Wasser im Bach fassen. Das Wissen um die Trasseeführung und die Appellativa und Namen der Suonen ist denn im Lötschental bei einer mittleren und jüngeren Generation erwartungsgemäss gering.

33 Freundl. Mitt. Gabriel Imboden; bestätigt: $d s$ Wasserleitji, Lina Zengaffinen, *1921, 02.2002; Simon Kalbermatter *1932, 03.2002. 
Generationen das Wässern nicht mehr selbst praktiziert haben, können sich künstliche Appellativa zuweilen durchsetzen. ${ }^{34 / 35}$

\section{$5 \quad$ Weitere Appellativa für Wasserleitungen}

Im Folgenden sollen die weiteren Wasserleitungsbezeichnungen in ihrer Wortbedeutung, Wortverbreitung und Etymologie erfasst werden. Semantisch unterscheiden sich die einzelnen Appellativa zumal durch die Grösse der Wasserleitung, die sie bezeichnen. Auch lässt sich von den Bezeichnungen meist ablesen, ob es sich um eine Wasserleitung am Beginn, in der Mitte oder am Ende der Strecke handelt. Aber es fällt nicht immer leicht, die Appellativa in ein System zu bringen, denn es verzweigen sich zwischen der Fassungsstelle beim natürlichen Wasser und dem Abflussgraben oft mannigfach auseinanderlaufende Kanäle.

\subsection{Die Hauptleitungen}

Die Hauptleitungen führen das Wässerwasser von der Fassungsstelle über eine lange Strecke hin zu den einzelnen Verteilern. Im Gegensatz zu den Verteilern, fliesst in Hauptleitungen permanent Wasser. Im ganzen Wallis gibt es laut Eichenberger (1940: 61) um die 300 Hauptwasserleitungen, die je einige Kilometer lang sind. Sie liefern ohne weiteres 200-300 Sekundenliter. Nebst den bereits behandelten Appellativen, Suon und Wasserleite, können sie mit sieben weiteren Appellativa benannt werden.

\subsubsection{Wasserfüer $\mathbf{f}$.}

\subsubsection{Lebendbelege}

Für Eggerberg belegt In-Albon Wasserfuhr: "Jede Hauptwasserleitung, jeder Rüüss, jede Zettwasserleita (aber nicht der Schrapf) konnte auch Wasserleita oder Wasserfüor genannt werden." (In-Albon 1997: 38).

Die acht Lebendbelege in den Dateien der Orts- und Flurnamensammlung Oberwallis verteilen sich auf vier in Salgesch und Varen, zwei in Mund, je einen in Naters und Feschel.

Einen Negativbeleg ergab die Nachfrage in Oberems (Wasserfüer sei ihm unbekannt, Raphael Biffiger, *1946).

\subsubsection{Historische Belege}

In den Walliser Landratsabschieden - vergleichbar mit den heutigen Protokollen des Kantonsparlamentes - taucht im Jahre 1537 "wasserfurungen" als Appellativum auf (Bd. 3: 151e). Der Name Wasserfuor ist 1666 belegt (Dateien der Orts- und Flurnamensammlung Oberwallis). Im 19. und 20. Jh. sind fassbar die "gippi Waserfuhr" (Tess. gib, gub 'Alpenrose' REW 4628a; mit Diminution wallis. -i) 1849 in Naters, die "Tenner Wasserfuehr" (vermutlich nach dem ON Tennen) 1859 in Eggerberg und die "Wasserfuhr" 1927 in Salgesch. Gegenüber den dreistelligen Zahlen von Wasserleiten- und Suonenbelegen fallen die wenigen Nennungen von Wasserfuhr deutlich ab.

\footnotetext{
$34 / 35$ Dieser Umbruch vollzog sich im Wallis verhältnismässig rasch. So berichtet etwa Werner Imseng aus SaasFee, wie das Wässern infolge des sich intensivierenden Tourismus seit 1951 (Strassenbau!) immer mehr zurückgegangen und bereits 20 Jahre später, 1973, Alois Burgener der einzige und letzte gewesen sei, der seine Wiesen bewässerte. Imseng (1993: 58).
} 


\subsubsection{Wortverbreitung und Wortbedeutung}

Laut Eichenberger (1940: 61) gehört Wasserfüer zu den Bezeichnungen für die grossen Hauptwasserleitungen; gemäss Idiotikon ist es synonym zu Suen (Id. 7: 1109). Verbreitung findet das komponierte Appellativum im Wallis, aber auch in Appenzell (Id. 1: 974).

\subsubsection{Etymologie}

Das Grundwort lautet schwzdt. gewöhnlich ohne Palatalisierung Fuer 'Beförderung von Waren und Personen auf der Achse oder zu Wasser, Führung' (Id. 1: 970f). Als Simplex ist es im Ahd. und Mhd. nicht belegt. Wohl Nomen actionis zu fuoren 'führen, leiten, ziehen'. ${ }^{36}$

\subsubsection{Höitfüeri f.}

Gemäss Eichenberger ebenfalls Appellativum für die grossen Hauptwasserleitungen (1940, 61). Zum Bestimmungswort: wallis. Hoid, Höit 'Haupt, Kopf' (Grichting 1999, 109), ahd./mhd. houbet 'Haupt, Kopf'. Unter der Verwendung als Bestimmungswort funktioniert Haupt- als Präfixoid mit der Bedeutung 'oberster, erster, wichtigster' (Kluge 1995, 360). Der Schwund von ahd. $b$ in wallis. Höit ist gemäss Bohnenberger für $b$ zwischen $o u$ und $t$ im Wallis die Regel (Bohnenberger 1913, 180).

Zum Hauptwort: ahd. *fuori Verbalabstraktum mit Suffix ahd. -i zu fuoren (jan-Verb) 'führen, leiten'. Schwzdt. Füeri 'einmalige Fahrt, ${ }^{37}$ Fuder'.

\subsubsection{Wärch n.}

Gemäss Eichenberger (1940: 61) ebenfalls Bezeichnung für die grossen Hauptwasserleitungen; sie erscheint aber in den Dateien der Orts- und Flurnamensammlung Oberwallis und in der Literatur nur selten.

\subsubsection{Lebendbeleg}

Niwwärch (das 'neue Werk') in Ausserberg.

\subsubsection{Etymologie}

Wallis. Wäärch 'Werk, Arbeit, Werg' (Grichting 1998: 235); ahd. werc(h) 'Werk, Tun, Tat, Arbeit, Ertrag'; mhd. werc(h) 'Werk, Tat, Handlung, Geschäft, Arbeit'. Verbalsubstantiv zu ahd. werkon 'arbeiten, handeln, wirken'. Es benennt sowohl die Tätigkeit als auch den Gegenstand, der durch die Handlung geschaffen wird. Ab dem 17. Jh. beginnt sich ein Bedeutungsschwerpunkt auf technische Betriebsanlagen zu richten (Bergwerk, Hüttenwerk, Walzwerk usw.) (Pfeiffer 1989: 1964).

\subsubsection{Wasser n. (vgl. auch 6.1)}

\subsubsection{Lebendbelege}

Die Dateien der Orts- und Flurnamensammlung Oberwallis enthalten gut 100 Lebendbelege, von "alpuwasser" (Simplon-Dorf) bis "wiudiwasser" (führt zum Ort Wildi).

\footnotetext{
36 Ahd. fuora (o-Stamm) 'Fahrt, Weg' ist vermutlich aus einem anderen Verb, nämlich aus varan 'fahren, gehen' gebildet. Semantisch dürften sich aber die Verbalabstrakta aus varan und fuoren im Lauf der Zeit gegenseitig beeinflusst haben (Kluge 1995: 290/Pfeiffer 1989: 485).

${ }^{37}$ Zur Bedeutung 'Fahrt' siehe Anm. 36.
} 


\subsubsection{Historische Belege}

Eine dreistellige Zahl urkundlicher Belege deckt das ganze Oberwallis ab; zeitlich reicht die Spanne von 1297 mit "aque que dicitur wilerwasser" ('Weilerwasser') in Naters und 1302 mit "aqua cui dicitur swellinwasser" (ahd. swelli 'Balken zum Hemmen, Schwellen des Wassers') in Stalden bis zum Jahr 1845 mit Bergwasser in Glis.

\subsubsection{Wortverbreitung und Wortbedeutung}

Die Lebendbelege sind über das ganze Oberwallis verstreut und reichen von Salgesch bis nach Oberwald, wobei das Goms und die Vispertäler die meisten Belege verzeichnen.

\subsubsection{Etymologie}

Ahd. wassar, mhd. wasser. Das mda. Appellativum bezeichnet nicht nur das Element, sondern auch Orte, wo sich Wasser befindet, natürliche Wasserstellen wie Quellen, Rinnsale, Bäche, Teiche usw. und auch Kulturanlagen, die Wasser führen, Rohrleitungen oder Bewässerungskanäle (FLNB 1999: 5/578).

\subsubsection{Rüüs m.}

\subsubsection{Lebendbelege}

Salgesch: Hier erscheint das Appellativum als Bestimmungswort eines komponierten Namens: Das Rüswasser ist die offene Wasserleitung vom Raffillji ins Dorf (Mathier 1989: 63).

Erschmatt: Rüss sei ein künstlicher Wassergraben, ein häufiger Ausdruck, auch ga rüssu 'das Laub und den Dreck aus dem Wassergraben entfernen' (Edmund Steiner, *1956).

Blatten/Lötschen: $\ddot{a}$ Ruis (m., Sg.) wie z.B. der Troselruis (Bestimmungswort vermutlich zum FlN $t s$ drosle $^{38}$ ) östlich Weissried hänge nicht notwendig mit Flurbewässerung zusammen, sondern meine primär eine natürliche Runse, durch die ein Bach hinunterfliesst, dann auch den Ort der Suonenfassung im Bachbett: "Inn Ruis geid mu gan d Suän aschlaan; ä Ruis ischt dr Bach, jedi Suän chunnd us äm Ruis." Wenn es geheissen habe: "dr Ruis ischt uisghiid" ('ausgefallen'), dann sei das Wasser bei der Fassung ausgebrochen und wieder in den Bach zurück geflossen. (Maria Bellwald-Siegen *1929, 02.2002).

Kippel: Als Namen bekannt sind der Hockuruis, der ob dem alten Hockenstafel ${ }^{39}$ aus dem Golmbach angeschlagen wird. Der Fururuis (Bestimmungswort Furu- zum FIN t fura) unter der Hockenalp speist sich aus dem Gafenbach; beide dienten zum Bewässern und zum Anwaschen ('Viehmist auf die Grundstücke ausschwemmen'). Appellativisch verstehe man unter einem Ruis einen Bach oder eine kleine Schlucht, durch die das Wasser hinunterfliesst; (Ignaz Bellwald, *1940, 03.2002).

In Ferden erscheint der Name Matturuis. Das ist der Wasserlauf, der den Berg hinunter durch den Faldumstafel und die darunterliegenden Faldummatten ${ }^{40}$ fliesst. (Papilloud 2000: 220).

In Oberems ist der Name Rüüss für den Hauptbach gebräuchlich. Es fliesse dort immer Wasser, und es handle sich um einen natürlichen Graben. Dementgegen steht hier die Wasserleitu, welche künstlich angelegt sei, kleiner sei und nicht immer Wasser führe. Das Verb rü̈̈ssu ("ds Wassr tüed rü̈̈ssu") meine ,graben, Land mitführen' und werde für kleine Erosionen gebraucht, während wüegätschu als nächsthöhere Stufe bereits einen eigentlichen Murgang meine. (Raphael Biffiger, *1946, 02.2002).

Steg: Mit Rüüs m., Pl. Rüssa, würden die im Talboden von der Lonza abgezweigten Gräben benannt, die ziemlich viel Wasser führen. Die Süenä dagegen seien am Berg. (Lina Zengaffinen, *1921, 02.2002).

\footnotetext{
38 Schwzdt. Drosel 'Bergerle' (Id. 10: 1764f.).

${ }^{39}$ Schwzdt. Hocke 'Sitz, Haufe' (Id. 2: 1120ff.).

40 Schwzdt. Matte w. 'Mähwiese, Wiese' (Id. 4: 548).
} 
Niedergesteln: $\ddot{a}$ Rü̈̈ss, Pl. Riiss, laufe immer senkrecht den Hang hinunter, ein kleiner Bach, der dann wieder eine (oder im Verlauf des Gefälles auch zwei, drei oder vier) Süe bzw. Süene speise (Dario Steiner, *1949, 10.2001)

Bürchen: $d r$ Rüss, selten äs Rüssji, sei ein steil herunterführendes Gewässer am Hang, "vellig es Wassr" (ein ziemliches Wasser) und grösser als eine übliche Wasserleite. Gemeinhin bezeichne Rüss eine Wasserleitung, indessen könne es sich zuweilen auch um einen natürlichen Bach, allenfalls mit leichten Korrekturen, handeln. (Oswald Zenhäusern, *1932, heute wohnhaft in Visp, 02.2002).

Grächen: ein Rüüs sei ein Wasserlauf, der entwässert: Der Rüüs gehe bereits durch das Dorf und führe unter dem Dorf senkrecht hinunter; wenn genug gewässert sei, werde das Wasser z Rü̈us gschlage (Reinhard Walter, *1939, 12.2001).

Saas Fee: Nicht als Appellativ für Bewässerungskanäle, sondern als Name für den Bach verwendet, der vom Hobalmgletscher herunterstürzt (Werner Imseng, *1924, 12.2001).

Eggerberg: "Nebenleitung der Hauptwasserleitung. Bei uns hat jede Hauptwasserleitung mehrere Rü̈̈sa. Jeder Hauptrü̈̈ss hat seinen Namen wie: (...)Winterrü̈̈ss, Halturü̈̈ss (Bestimmungswort Haltu vermutlich in Bezug zum FlN t halta ${ }^{41}$ ) etc." (In-Albon 1997: 35).

Birgisch: Russ, Name von der Wasserleitung der Heimmuhaltu, worein alle Nebenleitungen münden. (Dateien der Orts- und Flurnamensammlung Oberwallis).

Mund: Rüss als (künstlich angelegtes) Bächlein, wo ungebrauchtes Wasser hineingeleitet wird (Erwin Jossen 1989: 133).

Lax: Nicht benutztes Wasser werde über den Rottenschlag abgeleitet; "Der gleichbedeutende Ausdruck Rüss ist bei uns nicht bekannt" (Bielander 1941/42: 80 Anm. 2).

Geschinen: Das Wort ist unbekannt (Basil Werlen, *1932).

In der Literatur: Eichenberger (1940: 72) führt Rü̈̈s als Appellativum auf für Ableitungsgräben, die am unteren Ende eines Grundstückes auslaufendes Wasser aufnehmen (auch Pfandrüs und Zederrüs genannt).

\subsubsection{Historische Belege}

Ein früher historischer Beleg ausserhalb der Region stammt aus Klingnau/Aargau und datiert von 1267: "Aqueductum qui vulgariter dicitur runs, ..." (Id. 6: 1147). Für das Wallis nennen die Einträge in den Dateien der Orts- und Flurnamensammlung Oberwallis Archivbelege aus Eggerberg und Baltschieder (1424 "an dy runs), aus Raron (1433 "ad communem aqueductum cui dicitur antiquus Runs"), aus Geschinen (1520 "tendentem an den runss", 1674 "beym Russ", 1722 "Im Russ" und 1800 "bim Russ"; der Ausdruck ist den heutigen Gewährsleuten in der Gemeinde unbekannt) und aus Niedergesteln (1597 "Zettrunss").

\subsubsection{Wortverbreitung und Wortbedeutung}

Im Goms scheint der Ausdruck (vgl. Negativbeleg für Lax bei Bielander 1941/42: 80 Anm. 2 und negative Aussage für Geschinen) zumindest teilweise unbekannt zu sein. Als Verbreitungsgebiet nennt Zinsli (1946: 335) Bern, Graubünden, Glarus, Uri und Wallis. Innerhalb des Oberwallis resultiert aus der aktuellen Umfrage, dass der Begriff fast überall bekannt ist, dass aber die Bandbreite möglicher Bedeutungen so weit wie bei keinem anderen Begriff reicht. Sie variiert zwischen 'senkrecht verlaufende Hauptleitung' (Niedergesteln), 'natürliches Bachbett' 'herunterfliessender Bach' (Saas-Fee, Lötschen), 'Abflusskanal' (Grächen, Mund) und 'Wasserleitung' (Eggerberg, Salgesch). Grichting bringt 'Wasserfuhre' (Grichting 1999: 165) und das Idiotikon unterscheidet schwzdt. 1. 'starke und reissende Strömung' 2. 'Wasserlauf; Bach; Fluss' 3. 'Wildbach; Rinnsal; Wildbach, der nur während der Schneeschmelze fliesst' (Id. 6: 1142ff.), 4. 'Wasserrinne, Regenrinne, Strassengraben, Gosse' (Id. 6: 1148).

${ }^{41}$ Schwzdt. Halde/Halte 'Halde, Abhang' (Id. 2: 1174). 


\subsubsection{Etymologie}

Ahd. runs m./f. 'Flut, Wasser, fliessendes Wasser, Strömung, Wasserlauf', amhd. ruus (Zinsli 1946, 341). "eine auffällige s-Bildung" zu ahd. rinnan 'rinnen, fliessen'" (Kluge 1995: 335). Vgl. got. garuns. Der Schwund des $n$ mit Dehnung des Zwischenvokals entspricht dem Staubschen Lautgesetz. ${ }^{42}$ (Siehe auch Zinsli 1984: 579).

Eichenberger bringt für Salvan und Finhaut im frankophonen Teil des Wallis das Wasserleitungs-Appellativum rü m./n., Reynard für das Aostatal rus, das er von lat. rivus ableitet. Die Möglichkeit einer Entlehnung ist bisher nicht diskutiert worden.

\subsubsection{Härdt m.}

\subsubsection{Lebendbelege}

Reckingen: "Dr Härt isch das Wasser, ich hän dr Härt vam sibni bis em zwölfi"; hiess, ich habe Recht auf das Wasser von 7 bis 12 Uhr. (Hans Schmidt, *1927, Reckingen).

In der Gemeinde Reckingen gab es sieben grössere Wasserleiten, namentlich das Hauptwasser, das Oberwasser, das Tschupjiwasser, ${ }^{43}$ das Geishornwasser (Bestimmungswort Geishorn vermutlich in Beziehung zum FIN Geishore), Höfewasser (Bestimmungswort vermutlich zum FIN Hof), Bachbriggewasser ${ }^{44}$ und Schattenhalb. ${ }^{45}$ Als Appellativum für solche Wasserleitungen verwendete man Härdt (Reckingen 1995: 142f.).

\subsubsection{Wortbedeutung und Etymologie}

Schwzdt. Herd 'Erde' (Id. 2: 1597 ff.). Ahd. herd(a) 'Erdreich, Boden'. Vgl. Thomas Platter: "etlich wurzen waren ledig, das der herd an der gehen halden davon was gerissen" (Grimm 10: 1074). Herd ist im Oberwallis auch ein verbreiteter FIN.

Unser Härdt könnte die Erde bezeichnen, in welche die Wasserleitungen hineingegraben sind oder über welche sie hinwegführen. Möglicherweise ist auch die stützende Erdaufhäufung des Tretschbords angesprochen. Später wäre der Name der Erde auf die Wasserleitung übertragen worden. Die Wanderung eines Namens auf das benachbarte, umschliessende oder enthaltene Objekt kommt sehr häufig vor (vgl. z. B. Wüer).

\subsubsection{Wüer m.}

\subsubsection{Lebendbelege}

Steg: Dr Wicki-Wüer brachte das Wasser für Wickis Mühle (Mühle der Familie Wicky). Als dann die Lonza-Fabrik das Wasser für ihr Kraftwerk fasste und der Wüer kein Wasser mehr brachte, mussten sie ihm dafür gratis den Strom liefern. (Simon Kalbermatter, *1932, 03.2002).

Brig: Dr alti Wüer war der Bach, der das Wasserrad der Hammerschmiede betrieb (Fabian Perren, *1960).

Ernen: Der Wuhr, die mengenmässig grösste Wasserleite, von der andere abzweigten, führte Wasser zum Bewässern der Felder, Tränkwasser für das Vieh, Wasser zum Antrieb des Mühlrades (Carlen 2001: 72).

\footnotetext{
42 Bei Vokal + n + Reibelaut: n-Schwund mit Ersatzdehnung oder -diphtongierung des Vokals (Staub 1877/ Werlen 1977: 260).

${ }^{43}$ Wallis. Tschupji, Dim. zu wallis. Tschup 'Alpenrose'.

44, Bachbrückenwasser'.

45 'schattenseitig'.
} 


\subsubsection{Etymologie}

Ahd. wüer f., wuer m., mhd. wuor f. 'Damm im Wasser, Wehr zum Abhalten oder Ableiten des Wassers'.

Die Bezeichnung für das immanente Objekt (Wehr) geht über auf die Wasserleitung.

\subsection{Die Zuleitungen}

\subsubsection{Züeleita f.}

'Mittelgrosses Verbindungsglied zwischen den Bächen oder Hauptleitungen auf der einen Seite und den Feinverteilungskanälen auf der anderen'. Zusammengesetzt ist das Wort aus dem Verbalabstraktum leita (siehe 4.2.4) und einem präpositionalen Präfix - $z u$, das in palatalisierter Lautung erscheint.

\subsection{Die Feinverteilung}

\subsubsection{Schrapf $m$.}

\subsubsection{Lebendbelege}

Oberems: Dr Schrapf m., kleiner Graben, führe auf die Matte (Raphael Biffiger, *1946, 02.2002).

Niedergesteln: Ä Schrapf (ä Schrapfete Wasser) sei die Feinverteilung auf der Matte (Dario Steiner, *1949, 10.2001).

Eggerberg: "Die kleinste Verästelung von Wasserleitungen auf der Wiese" (In-Albon 1997: 35).

Grächen: Ein kleiner Ausläufer in den Grundstücken werde ebenfalls Schrapf genannt (Reinhard Walter, *1939, 10.2001).

Törbel und Zeneggen: "Ist in der Wiese eine Erhöhung, auf welche das Wasser nicht hingelangt, so wird eine kurze Wasserzuleitung (ein Schrapf) bis hierher gemacht, damit diese Stellen auch Wasser erhalten." (Stebler 1921: 83).

In Saas-Fee laut mündlicher Auskunft Werner Imseng (*1924, 12.2001) nicht bekannt.

Mund: Schrapf als kleinste Verästelung, wo das Wasser in die Wiesen geleitet wird (Jossen 1989: 133).

Lax: "Sollte das Gelände ohne Hilfsmittel nicht vom Wasser erreicht werden, so wird Abhilfe geschaffen durch das Wasserlädi ${ }^{46}$ oder durch einen Schräpf" (Bielander 1941/42: 103). "Der Schräpf ist eine kleine Querleitung auf die Matte hinaus." (Bielander 1941/42: 104).

Münster: Schrapf sei der kleinste Kanal auf die Wiese hinaus (Valentin Bacher, *1940, 02/2002).

\subsubsection{Wortbedeutung und Wortverbreitung}

Das Idiotikon schreibt: "'Stelle, wo ein kleiner Wassergraben von der Hauptleitung abzweigt'" (Id. 9: 1652). Auch wird Stebler (1921: 83) zitiert: 'eine gar kleine Wasserleite, die von einer grösseren herbeigeführt wird.' Unsere oben aufgeführten Belege weisen darauf hin, dass es sich um ein Appellativum für die kleinsten Ausläufer der Bewässerungskanäle handelt.

Ein Schrapf ist zuweilen nur ein bis zwei Meter lang und spült danach das Wasser fassungslos auf die Wiese hinaus. Solche kurzen Schräpfe werden in Reihen von sehr geringen Zwischenabständen (manchmal nur ca. zwei Meter) an die Zuleitung gelegt.

\subsubsection{Etymologie}

Es handelt sich um ein Verbalsubstantiv. Mhd. schrapfen '(Blut) schröpfen', frühnhdt. schrapfen ,abraspeln'. Germ. *skrapo 'raspeln, kratzen' (Kluge 1995: 742). Vgl. ahd. screfunga 'Einschnitt' und ahd. firscrofanen 'zerkleinert', wozu Raven ein ahd. *screfon konstruiert

\footnotetext{
${ }^{46}$ Ahd. latta/lata, mhd. late/latte 'Latte'; schwzdt. Ladi/Lädi 'Brett'.
} 
(Splett 1993: 857). Vgl. auch ahd. scrotan '(ab)schneiden, scheren, ausreissen, abziehen' (Seebold 1970: 425). Man führt in der Regel alle obigen Bildungen auf idg. *sker- 'schneiden' zurück (Seebold 1970: 742).

In der Terminologie des Gartenbaus bezeichnet schröpfen das Anschneiden von verhärteten Baumrinden, die neu belebt werden sollen. Der Schrapf ist also - analog zu den Einschnitten in Haut oder Baumrinde - eine Angrabung der Erde, um eine Wasserleitung anzuzapfen. (Siehe auch Id. 9: 1652).

\subsection{Die Abflüsse}

\subsubsection{Zetwasser $n$.}

\subsubsection{Lebendbeleg}

Erschmatt: "ds Wasser, wa unnena üsloift", sei das Zedwasser (Edmund Steiner, *1956)

Niedergesteln: ds zetwasser (Dario Steiner, *1949)

Grächen: "Zettwasser, das ischt ds Wasser, wa zundrescht üsa chunnd" (Reinhard Walter, *1939)

Visperterminen: "gang ab ga lüege, ob ds Zettwasser chund" (Gerold Vomsattel, *1964)

\subsubsection{Wortverbreitung und Wortbedeutung}

Das Zetwasser ist gemäss Eichenberger (1940: 89) das beim Wässern unten an der Wiese wieder auslaufende und in den Ableitungsrinnen aufgefangene Wasser. Der entsprechende Ableitungsgraben heisst Zedwasserleite, auch Zederrüs, Rüs, Pfandrüs (Eichenberger 1940: 72). Auch in Eggerberg ist das "Zettwasser ... jenes Wasser, welches (unten) aus der eigenen Wiese" fliesst bzw. die "Zettwasserleita" jene Wasserleite, die das Wasser am unteren Rand eines Grundstückes aufnimmt (In-Albon 1997: 39). Bielander (1940/42: 104) erwähnt für das unten abfliessende Wasser das Appellativum Zettete. ${ }^{47}$

Über die Qualität des Zettwassers kursieren gegenläufige Ansichten: Eichenberger (1940: 58f.) berichtet, es habe seine Nährstoffe auf dem Feld zurückgelassen und werde deshalb wenig geschätzt. Bielander (1940/42: 104) argumentiert entgegengesetzt: Das Zetwasser habe Mist (wallis. Büw) und andere Fettstoffe vom Feld aufgenommen und wirke deshalb jetzt wässernd und düngend zugleich. Wahr ist vermutlich beides: Die fruchtbaren Mineralien des Gletschers schlagen während des Wässerns auf dem Feld nieder, dagegen nimmt das Wässerwasser manche Nährstoffe, zumal den Mist, von der Wiese mit.

\subsubsection{Etymologie}

Wallis. zette 'zerstreuen, ausbreiten' (Grichting 1999: 246), schwzdt. zette 'zerteilen' (Eichenberger 1940: 89, Anm. 57); so wird etwa das frisch gemähte Gras auf der Matte oder dann das Heu in der Scheune zerzettet, also mit einer Gabel verteilt. Ahd./mhd. zetten 'ausstreuen, zerstreuen, verteilen'. Vgl. Uri und Davos: Zett-Gable,'Gerät zum Zetten des Heus' (Id. 2: 60), Erstfeld (Uri): PN Zettmist (Id. 4: 540), anord. tad 'ausgebreiteter Mist'.

Zet(t)wasser ist Kompositum mit Verbalstamm zet- als Bestimmungswort. Im Kontext des Bewässerns bedeutet (ein)zetten im Wallis: 'Eine Zuleitung dämmen, um das Wasser auf der Wiese zu verteilen'.

Vgl. in Ausserberg Zetti: 'in einem streifenweise bewässerten Grundstück einer dieser Streifen'. (Schmid 1994: 55).

\footnotetext{
47 Vgl. Wörter wie Gufrete, Chruitätä, Steinete, Gollätä oder Nesslätä. Feminine Bildungen mit Suffix -ete, ahd. -ata, vielleicht aus lat. Pl. -ata zu Sg. -atum. Das Kollektivsuffix wird oft zur Bildung von Wörtern verwendet, die ein massenhaft vorkommendes Nutzloses, Minderwertiges oder Gemischtes bezeichnen (Szadrowsky 1933: 77).
} 


\section{Die Eigennamen der Wasserleitungen}

\subsection{Namentypologie}

Es folgen einige der wichtigsten Eigennamen von Wasserleitungen, geordnet nach einer Namentypologie, die wir zu diesem Zweck aufgestellt haben.

\subsubsection{Ds Wasser}

Ein überaus häufiger Name künstlicher Bewässerungskanäle lautet $d s$ Wasser. Wenn es nicht als Simplex steht, so erscheint es als Grundwort eines Kompositums wie in alpwasser (Mund 1527 und 1726, Oberwald 1736 und 1737, Eggerberg 1774) oder tichellwasser (Oberems 1760, mda. Diichel, nhd. Teuchel 'Wasserleitungsröhre'). Zuweilen könnte es sich freilich auch um Schwundformen von Wasserleite handeln. In Ernen etwa wird dieselbe Leitung synchron Wichelwasser und Wichelwasserleite genannt (Carlen 2001: 72). Die Orts-und Flurnamensammlung Oberwallis zählt an die 150 Belege mit Wasser, wobei nicht in jedem Fall Bewässerungskanäle sondern manchmal auch natürliche Wasserläufe gemeint sind. Der Unterschied kann in der Regel nicht aus dem Namen heraus gelesen werden.

\subsubsection{Benennung nach Personennamen}

Die Benennung eines Bewässerungskanals nach einem Personen- oder Familiennamen kommt eher selten vor. Eine Wasserleite am Hang nördlich ob Salgesch heisst Bismarting (1494 "sub loz bis de martin", 1494 "aqueductu de martin", 1851 "biss Martin Wasserfuhr", "1880 Kehr im Bismarting"). Sie gehörte einem Martin oder führte durch dessen Güter (Mathier 1989: 49f.). Auf der Lauchernalp bauten Angehörige der Familie Senger um 1900 eine kleine Suon, ds Sengärsiändli. ${ }^{48}$ Für Baltschieder nennt Maurus Schmid die Martigo-Waserleita, die laut der mündlichen Überlieferung die ausgedehnten Güter der Familie Martig bewässerte bzw. als private Wasserleite von dieser Familie gebaut wurde; analog verzeichnet man in Mund eine Kienzleri ('Kienzlerin'); der Name Kienzler soll hier im 16./17. Jahrhundert belegt sein (Schmid 1994: 130/167).

Oftmals ist es schwierig, abzuschätzen, ob eine Leitung nach einem PN oder einem ON/FIN benannt ist. Die Seebärgärsuän zum Beispiel bezieht sich auf eine entsprechend benannte Landparzelle und nicht primär auf den Namen der Familie Seeberger, welche in der Gemeinde Kippel ansässig ist. "Gang die Suän gan meejen" meint in einem solchen Zusammenhang 'geh dieses Stück Land mähen'.

\subsubsection{Benennung nach Ortsnamen}

Sehr häufig sind die Wasserleitungen nach Orts- und Flurnamen benannt. Bei den entsprechenden Orten handelt es sich zumeist um

1. die Herkunft der Suän (Ort der Schöpfe): Das historische "gletscherwasser" (1305) in Grächen wird vom Riedgletscher hergeführt; das in Stalden 1691 belegte "bynden wasser" bezieht sich auf den FIN in der Binden und in Lax fliesst das Bettmerwasser, das aus der Alpe von Betten kommt (Bielander 1941/42: 80). ${ }^{49}$

2. den Ziel- oder Endpunkt der Suän (Ort des Auslaufens im Gelände): Ts furewaser in Blitzingen zur Bewässerung einer Flur namens Fura. Die Eggeri führt zum Weiler Egga

\footnotetext{
48 Freundl. Mitt. Ignaz Bellwald, Kippel, 03/2002. Vgl. Papilloud (2000: 218).

${ }^{49}$ Unsicher ist die Etymologie der Maanerra. Maurus Schmid meint, der Name leite sich entweder vom Maachii ('Maa-Schlucht') her, aus welcher die Wasserleite herausführt, oder aber er würde sich auf den Heiligen St. German beziehen und hätte ursprünglich Germaneri geheissen.
} 
(Grächen), die Chilcheri zum Dorfteil, wo die Pfarrkirche der einstigen Weilersiedlungen steht (Grächen), die Gorperi (Eggerberg) in den einstmals bewohnten Weiler Gorbji (InAlbon 1997: 8) und die Putzera in Ernen zum Putz, ${ }^{50}$ der als Viehtränke genutzt wird.

Wie schon unter 6.1.2 erläutert, ist es oft schwierig zu entscheiden, ob eine Leitung nach einem Ort oder nach einer Person benannt ist. So kann z. B. die Brigeri (1390 "in aqueductu qui dicitur superior brigarra"; 1972 "t ober brigeri") die Leitung der Briger oder die nach Brig führende sein. Manchmal können selbst einheimische Kenner sich nicht entscheiden. So führt in Grächen die Bineri das meiste Wasser zum Weiler Bina (1 km nördlich des heutigen Siedlungszentrums). Dort sind auch die meisten Geteilen ('Genossenschafter') der Wasserleite ansässig. Es könnte sich also um 'die Wasserleitung der Biner' oder um 'die Wasserleitung, welche nach Bina führt' handeln.

\subsubsection{Benennung nach einem räumlichen Bezug zu anderen Wasserleiten}

In Grächen gibt es ein vorders Wasser und ein hinders Wasser (ein südlicher und ein nördlicher durchfliessendes). Häufig anzutreffen im Wallis ist die Bezeichnung oberi, unteri, mittleri Wasserleita (oder Suon). Für Martisberg ist 1633 "das ausser wasser" bezeugt, für Betten 1763: "im obren Wasser".

\subsubsection{Benennung nach dem Alter}

Die Altersbestimmung durch Namen beschränkt sich zumeist auf die Unterscheidung von alten und jungen Leitungen. 'Junge' Leitungen sind: D Niwa ('die Neue') in Mund, das 1381 erstmals urkundlich erwähnte Niwwärch ('das neue Werk') in Ausserberg oder die angeblich schon um 1300 erwähnte Niwa aus dem Bietschtal (Raron, St. German; Schmid 1994: 101). Der verschwundene Name $d$ ober Niwwa aus Grächen könnte gemäss der Vermutung Reinhard Walters die Driijeri meinen (kommt aus einem Schöpfkasten mit drei Öffnungen), welche infolge des vorrückenden Riedgletschers versetzt werden musste. Um eine als (sehr) alt erachtete Leitung handelt es sich beim 1611 bekundeten "Heyden wasser" in Visperterminen.

\subsubsection{Benennung nach Grösse oder Beschaffenheit}

Di Grossu ${ }^{51}$ in Niedergesteln hat von allen Suonen der Gemeinde das grösste Fassungsvermögen. T hee Suän ('die hohe Suon') zwischen Wiler und Ried im Lötschental war beim Breiti Schleif in den Wüstenmatten so tief, dass ein Erwachsener bis auf Achselhöhe hineinstehen konnte. D Chänälsuän ist in Ried/Lötschen die einzige mit einem Teilstück aus Holz, alle anderen verlaufen im Erdreich; gleich verhält es sich mit dem Chänilwasser in Eggerberg (In-Albon 1997: 28) und dem Chänilwasser ob St.German und Ausserberg (Schmid 1994: 95). Die Grächer Driijeri hat in der Archa ('Schöpfkasten') drei Öffnungen mit Rickerlini ('Schieber'), gewöhnlich sind es nur zwei (Reinhard Walter, *1939, 12.2001). In Ausserberg fliesst $d s$ Horugiegi (wallis. Horu 'Horn, Berg'; Giegi, wallis. Guogo 'Wurm, Gewürm'52 mit ahd. Diminutiv-Suffix -i(n)); von über 2600 Metern Höhe über Meer mäandert die Leitung wie eine Schlange von Rotu Chue nach Leiggern und Raaft hinunter (Schmid 1994: $135)$.

\footnotetext{
50 Wallis. 'Teich' (Grichting 1999: 157); ahd. puzz 'Brunnen, Pfütze'.

51 Schmid (1994: 82) nennt sie die Grossá. Angeschlagen wird sie am Ort Chäli in der Schlucht ob dem Dorf, Auskunft Dario Steiner (*1949, Niedergesteln).

52 Id. 2: 159 f.; Schmeller bringt zum Vergleich span. cuca 'Wurm'. Bayrisch Gueg bedeutet 'Baumwanze' (Schmeller 1966: 881). Im Schwzdt. ist die Baumwanze der Gauch (Id. 2: 881), hergeleitet von ahd. gouh 'Kuckuck' (Kluge 1989: 247). Die Bedeutung begreift also stets minder geschätzte Tiere.
} 


\subsubsection{Benennung nach der Wasserqualität}

Die Sandwasserleita in Niedergesteln führt viel Sand mit sich (Schmid 1994: 82). In der Wissa in Lax und der Fiescherwiissa in Fiesch (1411 "wis wasserleita", 1451 "album aqueductum", 1532 "wysswasserleyten", 1588 "wisses wasser", 1681 "die wysa") fliesst Weisswasser vom Fieschergletscher herab. Das nah des Gletschers noch milchig trübe Wasser soll das Kleewachstum fördern (Bielander 1941/42: 80f.). Seine Farbe und Wirkung erhält es von feinem Granitsand und Schlamm (Stebler 1914: 64), der Littu. Man sehe diese noch als weisslichen Niederschlag auf den Feldern, welche von Gletscherwasser bewässert worden sind (Eichenberger 1940: 88). ${ }^{53}$

\subsection{Besonderheiten}

\subsubsection{Namenlose Wasserleiten}

Nicht jede Suon hat einen Namen. "Di in dr Bärgmattun, wa vam Jungschten aha chunnd, di hed kchein Namm." ('Die in der Bergmatte, welche vom Jungschten herabfliesst, trägt keinen Namen.') (Karl Bellwald, *1926, Blatten/Lötschen 10.2001). Generell keine Namen tragen die Suonen auf der Kummenalp und der Restialp. Sie wurden, wo überhaupt, $d$ Alpusuän genannt. ${ }^{54}$

Für die Gemeinde Hohtenn schreibt Schmid, dass zwei namenlose Wasserkanäle aus dem Jolital führen, die obere und die untere Namenlose (Schmid 1994: 73) - hier gerieten die Namen der längst abgegangenen Leitungen in Vergessenheit oder wurden eventuell auf die neueren Kanäle übertragen.

Daneben gehen heute manche Wasserleitungsnamen, wie die Flurnamen überhaupt, verloren; selbst die ältere Generation muss zuweilen länger nachdenken, um sich bestimmter Namen zu erinnern, und oft fällt dabei die Bemerkung: ja, ich war halt schon Jahre nicht mehr an jenem Platz; früher, als wir noch Landwirtschaft hatten, da mussten wir oft genug dorthin und da kannten wir die Namen. "Us im Troslruis iwärd geid ä Suän, abr wiä heissid dii? Ah, di hed appa kchein Namm." ('Aus dem Troslrüs wird eine Suon abgeleitet, aber wie heisst die noch? Nun, die wird wohl keinen Namen besitzen.') (Cäcilia Siegen, *1933, Ried/Lö, 11.2001). Man kann wohl von einer grossen Zahl urkundlich nicht erfasster und vergessener alter Leitungsnamen ausgehen. Eine diachrone Charakteristik der Suonen verbleibt deshalb immer trümmerhaft.

\subsubsection{Nach Abschnitten wechselnde Wasserleitungsnamen}

Manche Bewässerungskanäle ändern ihren Namen im Verlauf der Strecke. So wird die Tatzsüe (Bestimmungswort vermutlich in Bezug zum Weiler Tatz), nachdem sie einen Rüüs hinuntergestürzt ist, zur Ladesüe (fliesst durch Ladu'55) in Niedergesteln; die Brägjeri führt nach dem Brägji (Weiler ob Niedergesteln) und heisst später Thelsüe (Bestimmungswort Thel steht vermutlich in Bezug zum FIN Telwald ${ }^{56}$ ). In Mund heisst der letzte Teil des Chriizwassers (Bestimmungswort Kreuz vermutlich mit Bezug zum FIN Chriiz) die Schüfleri (vermutlich in Bezug zum historischen FIN "schuflun"57), die Wyssa hiess bis zum Ort Finnen die

\footnotetext{
53 In Hohtenn sagt man, der Reub ('das Heu') am Innru Wasser ('am Inneren Wasser'), das Gletscherwasser führt, sei zu mähen wie Salat (Schmid 1994: 71).

${ }^{54}$ Freundl. Mitt. Ignaz Bellwald, Kippel, 03/2002. Vgl. Papilloud (2000: 221).

55 Wallis. Lade m. 'Brett' (Grichting 1999: 125); mhd. lade 'm. 'Brett, Bohle'.

56 Wallis. tela f., rom. dalia 'Föhre' (Zimmermann 1968: 26).

57 Ahd. scuvala, schuvel; mhd. schufel 'Schaufel'.
} 
Wichieri (vermutlich zum historischen FIN "in den Wichjen"58). Wenn die Äbineri (fliesst durch die Flur Ebine) in Eggerberg den Ort Schliecht passiert hat, heisst sie fortan Tenneri (vermutlich in Bezug zum ON Tenn; Schmid 1994: 178, 167 und 150). Im Goms heisst eine Wasserleitung in Niederwald vom Walibach bis in den Brand di gmei Wasserleita ('öffentliche Wasserleitung/Gemeindewasserleitung'), in Selkingen und Blitzingen aber $d$ Walderi (wird am Walibach gefasst). Laut Manfred Mathier wird die Tschampichtruwasserleite (Bestimmungswort in Bezug zum FIN Tschampichtru ${ }^{59}$ ) in Salgesch von der Fassungsstelle bis zum Schiessstand Binüt genannt (gemäss Mathier 1989: 49 möglicherweise aus bis und afrprov. nue, also 'neue Wasserleitung'). Das Mengis ${ }^{60}$ nennt sich ab Tschangerang Bisetji (mit Doppeldiminutiv: frz. - et und wallis. -ji; Mathier 1989: 54).

Dagegen finden sich auch sehr lange Wasserleitungen, die ihren Namen nicht ändern. So die Chaschtlärra (fliesst nach Kastel) in Lötschen: Sie durchfliesst vier Gemeindeterritorien und eine Vielzahl von Fluren, ändert aber ihren Namen während der ganzen ca. 10 Kilometer langen Strecke nicht.

\subsubsection{Suonen mit zwei oder drei Namen}

Stebler $(1921,71)$ nennt für Törbel/Zeneggen eine Leitung mit drei Namen: Das Augstbordwasser (wird am Augstbordbach in der Gemeinde Embd gefasst) die Augstborderin, die Niwe. In Mund hiess man das Dorfwasser auch Wylerwasser (Jossen 1989: 134 und 139). Die Siedlung, durch welche das Wasser floss, sei nämlich bis ins ausgehende 18. Jahrhundert auch Wyler genannt worden (Schmid 1994: 174). Es handelt sich hier um Doppel- und Dreifachbenennungen, deren Motiv im einzelnen abzuklären bleibt. ${ }^{61}$

\subsubsection{Namenwechsel}

Die Dateien der Orts- und Flurnamensammlung Oberwallis enthalten nur ein Beispiel, das von einem historischen Namenwechsel zeugt: Das Brunniwasser (fliesst vom Ort Brunni her) in der Gemeinde Martisberg hiess früher ts Klotzwasser (Klotz 'Wasserfassungskasten'). Für Grächen berichtet Reinhard Walter (mündliche Mitteilung 11. 2001), dass die Eggeri, die heute bis zum Weiler Egga reicht, einst über das Bärgji bis Illas gegenüber Stalden führte. Die Wasserleite hiess daher Eggeri und Bärgeri, wie auch Alois Larry Schnidrig schreibe. ${ }^{62}$

Exemplarisch für den heutigen Umgang mit dem Namengut ist folgende Begebenheit: Ignaz Bellwald, Lokalforscher im Lötschental, eruierte für das ganze Tal über 150 Suonen. Da die Suonen auf der Resti- und in der Kummenalp beide d Alpusuän heissen, taufte sie Ignaz Bellwald zum Zweck der Unterscheidung $d$ Reschtialpsuän und $d$ Chummunalpsuän (Ignaz Bellwald, 11.2001). Dieses Beispiel eines Namenwechsels in neuester Zeit fand mittlerweile in die Literatur Eingang. ${ }^{63}$

\footnotetext{
${ }^{58}$ Gemeindearchiv Glis, B. 8c. S. 31. Eggerberg 1859.

${ }^{59}$ Lat. in campestris 'in der Ebene' (Mathier 1989).

${ }^{60}$ Gemäss Mathier aus lat. magnus und gall. *bedu 'grosse bisse'. Vielleicht wäre auch die Herleitung vom FaN Mengis in Salgesch, der seit 1820 in Leuk und Visp bezeugt ist, möglich.

${ }^{61}$ Gelegentlich kommt es auch zu Missverständnissen: Die längste Suon des Lötschentales, die von Ried-Blatten über alle vier Talgemeinden bis nach Kastel gegenüber Ferden führt, heisst $t$ Chaschtlärra wird aber von einigen Gewährsleuten fälschlicherweise auch thee Suän genannt. Der Irrtum entsteht laut Erklärung von Ignaz Bellwald dadurch, dass beide Suonen am selben Ort gefasst und über eine erste Strecke gemeinsam geführt werden, sich dann aber in die eigentliche Chaschtlärra und in die hee Suän verzweigen.

${ }^{62}$ Reinhard Walter sagt, er habe selber noch das Tretschbord in der Felswand bei Hohtschuggo gesehen.

63 Ignaz Bellwald (*1940, Kippel) hatte 1997 im Hinblick auf die Publikation "Die Suonen des Wallis", erschienen 2000, die Namen von allen künstlichen Bewässerungskanälen in der Talschaft Lötschen zusammengestellt (inkl. Unteres Taldrittel bis Steg/Gampel). Die Kummenalp-Suon und die Restialp-Suon befinden sich in der Publikation auf den Seiten 220 und 221.
} 


\title{
6.3 Morphologie der Wasserleitungsnamen
}

Kompositiv werden die Namen gebildet, indem einem appellativischen Grundwort für Wasserleitung (Suon, Wasser, Wärch, Wasserleita usw.) ein Bestimmungswort vorgestellt wird, das Orte, Situation, Besitzernamen, Alter, Beschaffenheit oder Wasserqualität der Wasserleitung anzeigt. In der überwiegenden Zahl handelt es sich um Zusammensetzungen zweier Substantive (z. B. Gletscherwasser), die in der Regel zu eigentlichen Komposita verbunden sind, d. h. zu Verbindungen, deren Bestimmungswort in der Stammform steht. Auch in den Verbindungen Adj. + Subst. (z. B. Oberwasser) und Verb + Subst. (z. B. Zedwasserleite) verbleibt das Bestimmungswort zumeist in der Stammform. Eine syntaktische Fügung findet sich beim Namen $T$ hee Suon.

Bei den Simplexformen herrschen substantivierte Adjektiva (z. B. Wissa) und movierte ONAbleitungen mit dem Suffix -eri (z. B. Eggeri) oder -ar(r)a (z. B. "gornarra") vor. Das alte Suffix - $\operatorname{ar}(r) a$ (Fem. zu ahd. - ari) findet sich aber nur in historischen Belegen. Es wird später zu - eri (aus ahd. Personalsuffix -ari + movierendem Suffix -in(na)), so die "brigarra" (1390) > Brigeri, oder die "gornarra" (1390) > Gorneri. Diminution kommt bei den Wasserleitungsnamen weniger häufig vor (z. B. Horugiegi), ${ }^{64}$ ebenso der Simplex in Stammform (z. B. Wasser).

\section{$7 \quad$ Appellativa für Konstruktionen, Elemente und Werkzeuge}

Zuletzt soll noch ein kleiner Überblick über die wichtigsten Appellativa von Konstruktionen und Werkzeugen sowie von Tätigkeiten im Gesichtskreis der Wasserleitungen gegeben werden.

\begin{abstract}
Abschlaga f., Abschlacht f.
'Umleitungsschleuse in der Wasserleite' (Bielander 1941/42: 101f.). Gemäss Eichenberger auch 'Fassungsstelle' (Eichenberger 1940: 79). Das Wasser abschlagen bedeutet, die Schleusen so zu stellen, dass das Wasser wieder entlang des Hauptkanals oder des Abzugskanals in das Tal fliesst. (Eichenberger 1940: 87). Die Abschlaga ist nach dem Abschlagen des Wassers benannt, jedoch dient sie auch der entgegengesetzten Tätigkeit, dem Einleiten des Wassers in die Zuleitungen. Ahd. slaga, mhd. slage 'Hammer, Schlag, Spur vom Hufschlag, Fährte'. Fem. Nomen actionis (o-Stamm) zu ahd. slahan, mhd. slahen. Ahd. abaslahan, mhd. abeslahen 'abschlagen, beschneiden'. Vgl. Schwzdt. Abschlag 'Ableitung eines Wasserlaufs, Stelle bei einer Wasserableitung, Abzugsgraben' (Id. 9: 198). Die Bedeutungsentwicklung genau zurückzuverfolgen gestaltet sich, wie bei Aschlag, auch hier schwierig.
\end{abstract}

\section{Anschalte f.}

'Wasserfassung am Bach' (Eichenberger 1940: 79). Ahd. scalta, mhd. schalte 'Stange zum Fortstossen des Schiffs', fem. Nomen actionis (o-Stamm) zu ahd. scaltan, mhd. schalten '(ein Wasserfahrzeug) fortschieben, entfernen'. Ahd. anascaltan 'ins Wasser/Meer schieben'. Schwzdt. aschalte 'ein Schiff durch Stossen in Gang setzen, abstossen' (Id. 8: 714). Vgl. Wallis., grbnd. Abschalte 'Abzugskanal' (Id. 8: 710). Wallis., aarg., grbnd. abschalten 'fliessendes Wasser ablenken, ableiten', sonst schwzdt. 'etwas wegschieben, -stossen' (Id. 8: 714).

Das Wort dürfte also die 'Entfernung und Ableitung des Bachwassers' bezeichnet haben, um dann in der Bedeutung überzugehen auf das notwendige Instrument hierzu. ${ }^{65}$

\footnotetext{
64 Weitere Beispiele: In Ried-Brig ts Eischterli (Wasserleite vom Ort Eistlin oder Eysterlin; 1389 "in aqueductu qui dicitur oystwasserleita", 1700 "cum...medio opere aus dem Eysterlin"), in Glis ts Oberli (Wasserleite beim Ort Holzji) und in Termen ebenfalls ts Oberli (verfallene Wasserleitung im Gartwald).

65 "Nomina actionis, die dann gern als Gegenstandsnamen auf konkrete Geleise geraten" (Henzen 1947: 131).
} 
Vgl. als Gegenstück zur Anschalte die Abschalte. Weil dieselbe Konstruktion, nämlich die Wasserfassung, das Bachwasser in die Wasserleitung einlässt oder den Zugang sperrt, sind Aschalte und Abschalte de facto Synonyme (Eichenberger 1944: 77).

\section{Aschlag m., Aschlacht f.}

'Wasserfassung am Bach' (In-Albon 1997: 13). Ahd./mhd slag 'Schlag, Schlag mit der Hand oder einem Instrument'. Mask. Nomen actionis (i-Stamm) zu ahd. slahan, mhd. slahen 'schlagen, einschlagen auf'. Ahd. anaslahan '(dar)anschlagen, anbranden'. Vgl. schwzdt. Abschlag 'Ableitung eines Wasserlaufs, Stelle bei einer Wasserableitung, Abzugsgraben'.

Die semantische Entwicklung von slag ist sehr vielfältig und die Begründung von einzelnen Bedeutungen deshalb oft schwierig zu leisten (Id. 9: 197f.). Könnte ursprünglich gemeint sein 'das Schlagen/Hacken/Graben eines Kanals an das Bachbett'?

Der Rottenschlag ist der Aus- und Ablauf einer Wasserleitung in die Rhone (Rotten). (Bielander 1941/42: 80).

\section{Bänne f.}

'Holzwasserleitung aus zusammengenagelten Brettern' (Eichenberger 1940: 72f.). 'Schwzdt. Benne 'aus Brettern zusammengesetzter, beweglicher offener Kasten, angebracht auf einem Wagen oder Schlitten, um darin Dünger, Erde, Sand, Kartoffeln und Obst fortzuschaffen' (Id. 4: 1289f.). Lehnwort entweder aus dem Gallischen oder Römischen (Id. 4: ibid.). Gall./röm. benna 'Korbwagen' (REW: 89).

Mit dem Benennen der bretternen Walliser Wasserleitungen wurde das Bild eines Kastens evoziert, was den Unterschied markierte zu den Leitungen aus ausgehöhlten Baumstämmen. Allerdings bringt das Idiotikon Benne auch als Walliser Appellativ für ausgehöhlte Baumstämme, die als Brunnentröge dienen (Id. 4: ibid.).

\section{Chänäl m.}

'Holz-, Eisen- oder Zementwasserleitung im Unterschied zu den Grabenleitungen' (Eichenberger 1940: 72f.). Bei den Holzleitungen handelt es sich sowohl um ausgehöhlte Tannenund Lärchenstämme als auch um Leitungen, die aus Brettern zusammengesetzt sind. Schwzdt. Chännel 'künstliche Rinne zur Fortleitung des Wassers, besonders Dachrinne' (Id. 3: 310f.). Ahd. kanali, chanali, kanil, kenil, mhd. kenel 'Kanal, Rinne, Gosse, Röhre'. Lehnwort aus lat. canalis 'Röhre, Rinne, Wasserlauf', einer Substantivierung von lat. canalis m./f. 'Rohr, Rinne, Graben, Kanal'.

Im Italienischen wurde lat. canalis zu canale und wanderte ein zweites Mal ins Deutsche: nhd. Kanal (ab 15. Jh.). Dagegen verweisen in Chänel die k-Verschiebung und der Umlaut auf die frühere Entlehnung in ahd. Zeit.

Für Lax nennt Bielander (1941/42: 86 und 91) Sg. Chännu und Pl. Chänja, für Ausserberg: $d$ Chännja. Gemäss In Albon verstand man in Eggerberg unter Chänäl "früher nur ausgehöhlte Baumstämme, später selten auch drei Bretter, rechtwinklig mit Nägeln aneinander befestigt" (In Albon 1997: 32).

\section{Glotz m.}

In Lax: ,Hölzerne oder eiserne Schleuse mit regulierbarem Massrahmen' (Bielander 1941/42: 85 Anm. 2). Glotz ist ein charakteristisches wallis. Appellativum: 'Luke, (viereckiges) Guckloch, Seelenfenster, Auge' (Grichting 1999: 89). Wallis. gglotzu 'schauen, sehen'. Das Verb ist erst seit dem 13. Jh. bezeugt, mhd. glotzen 'mit weit aufgesperrten Augen blicken, starr blicken, stieren'. 
Die Herkunft des Wortes ist bisher nicht ermittelt worden. Vgl. mittelenglisch glouten 'starren' und anord. glotta 'grinsen' (Kluge 1995: 328).

Benannt werden stets Öffnungen: Auge und Fenster (sowie bei dem zum Vergleich herangezogenen Verb glouten das Öffnen des Munds). Glotz ist vermutlich ein Nomen actionis, das die Öffnung der Schleuse bezeichnet hatte. Von hier wäre das Appellativum übertragen worden auf die gesamte Schleusenanlage.

\section{Gufr m./n.}

'Wässerstein', s.a. Wässrblatta. Stein mit der Dämmfunktion von Wässerplatten. Am besten eignen sich Schieferplatten von höchstens $10 \mathrm{~cm}$ Dicke (Eichenberger 1940: 92). Berner Oberland, Uri, Graubünden und Wallis: Ggufer, Ggufr 'kleinerer Felsblock, Stein, Kieselstein, Steinhaufen, Geröll' (Id. 2: 132). Die Etymologie ist rätselhaft. Prov. findet sich combre 'Schutthaufen', woraus sich mhd. kumber herleitet, aus dem das nhd. Kummer wird (wohl als metaphorische Übertragung). Man konstruiert dazu ein gall. *komboros. Da in der Wortentwicklung von *komboros zu kumber und Kummer der Nasal erhalten bleibt und ein Labiodental nicht auftaucht, lässt sich *komboros nicht leicht als Wurzelwort zu unserem Gufr setzen. Hubschmied versucht gleichwohl einen Lautwandel von $m b$ zu $f$ zu begründen, damit er die These eines gallischen Substrats durchsetzen kann. Es habe sich im Spätgallischen aus *komboros ein koexistierendes *kombro entwickelt, das zu *kovro und *kuvro geworden sei (Hubschmied 1938: 133ff.). Belegbare Hinweise, die auf einen solchen Wandel schliessen lassen würden, gibt es nicht. Scheuermeier bestreitet deshalb Hubschmieds Etymologie und verweist auf romanisch Cubulum 'Lagerstätte des Viehs' zu lat. cubare 'ruhen'. Solche Stätten fanden sich oft in Höhlen unter überhängenden Felsen, schwzdt. Gufel 'Chobel 'Felshöhlung als Unterkunft für das Vieh, Höhle' (Id. 2: 132). Sodann müsste man eine Übertragung des Appellativums für 'Höhle' auf den Fels und Stein annehmen. (Scheuermeier 1920; Pokorny VR 10: 242; Zinsli 1984: 568).

\section{Howa f.}

'Hacke', s.a. Wässerbiel. Schwzdt. Hauwe 'Hacke mit Schneide zur Arbeit in Feld, Garten und Weinberg' (Id. 2: 1801ff.). Ahd. howa, houwa, mhd. houwe 'Haue, Hacke'. Nomen actionis (o-Stamm) zu ahd. houwan, hauwan, mhd. houwen 'hauen'.

Bielander bringt (1941/42: 95) Dim. ds Heuwji und ds Schorrheuwji.

\section{Sanderna f.}

'Sandfang' (Eichenberger 1940: 79). Das Suffix -ernen ist eine Verbindung von lat. -arius, -a, -um mit westgerm. -injo: zunächst vereinen sie sich zu -ar-in, daraus wird -erina, im Nom. Pl. -erinen, mit Schwund des Inlautvokals: -ernen (Schnetz 1952: 14f.). Das Suffix war natürlich besonders an der romanischen Sprachgrenze produktiv, mit ausserordentlicher Häufung auf dem heute deutschschweizerischen Gebiet.

Sanderna: 'Ort mit (viel) Sand', d. i. in unserem Fall der Sandfang. Gemeinhin ist das Fangen des Sandes eine Nebenwirkung der Wasserfassung. Der Sand wird hier gedämmt durch die Schleuse. Aus diesem Grund fungiert Sanderna oft als Synonym für Aschlag, Aschepfi usw. (Eichenberger 1940: 79).

Bei Bielander erfahren wir aber noch von einer weiteren Möglichkeit, den Sand zu fassen. Für Lax beschreibt er "Ausbauchungen und Ausbuchtungen der Wasserrinne, worin ebenfalls der Sand zurückbleibt"; Sie werden "in gewissen Abständen in die Wasserleite eingebaut", dort wo das Wasser die Privatgüter noch nicht erreicht hat. Periodisch muss die Sanderna von den Gmeiwärchern ('Gemeindearbeiter' = 'Gemeindemitglieder, die genossenschaftliche Arbeit verrichten') geleert und gereinigt werden (Bielander 1941/42: 91). 


\section{Sapa f.}

'Wässerbeil'. Charakteristisches Gerät, um Wassergräben auszuheben. Auf der einen Seite ist es mit einer Schneide, auf der anderen mit einem Bart versehen (Eichenberger 1940: 90; Id. 7: 1221). Schwzdt. Verb sappe 'einen Stein, einen Baum mittels eines Hebels aus dem Boden herauswägen' (Id. 7: 1221). Nhdt. in der Militärsprache Sappe 'Unterminierungsgraben, Laufgraben in Richtung auf die feindlichen Stellungen, Belagerungsgraben' und sappiren 'unterminieren'. Es handelt sich um ein neueres Lehnwort: frz. sape ,Unterminierungsgraben', frz. saper 'unterminieren, eine Mauer untergraben'. Die weitere Herkunft ist unklar (Kluge 1995: 704).

\section{Schepfi f.}

'Wasserfassung' (Eichenberger 1944: 79). Schwzdt. Schepfi 'Ort, wo man Wasser schöpft' (Id. 8: 1052f.). Ahd. scepfi in scepfi-faz 'Schöpfgefäss, Kelle'. scepfi ist Verbalabstraktum mit Suffix ahd. -i zu ahd. (gi)scepfen 'schöpfen' (jan-Verb); die Bedeutung wäre in unserem Fall: ,Ort wo geschöpft wird'.

\section{Schwellebank m.}

'Wasserfassung' (Eichenberger 1940: 79). Zum Grundwort: Schwzdt. Bank 'Sitzbank, Ablegegestell' (Id. 4, 1380)' 'Geländeterrasse' (Zinsli 1946: 312). Ahd. bank, mhd. banc 'Bank, Tisch'. Vgl. anord. bakki 'Erhöhung'. In unserem Fall vielleicht metaphorische Benennung des Fassungskastens.

Zum Bestimmungswort: Ahd. swella, mhd. swelle 'Schwelle, Balken zum hemmen, schwellen des Wassers', Verbalnomen zu ahd. swellan, mhd. swellen 'schwellen'. Auch: Schwellibritt, Schwellbaich.

\section{Schwälligufr m./n.}

'Wässerstein' (Eichenberger 1940: 92). Zum Grundwort siehe Gufr.

Wallis. Schwelli, Schwälli f. (Grichting 1998: 187) auch 'grosse Flüssigkeitsmenge'; ahd. swelli $\mathrm{n}$. 'Schwelle, Balken, der schwellen macht, der das Wasser hemmt und abhält', zu ahd. swellan, mhd. swellen 'anschwellen'.

\section{Schwärbe}

'Wasserfassung' (Eichenberger 1940: 79). Verbalnomen. Ahd. Verb swerban, swerpan 'wischen, abreiben, abtrocknen, mhd. swerben 'schnell hin- und herfahren, sich verwirrt oder wirbelnd bewegen, schwirbeln, wirbeln'. Schwzdt. schwerbe 'fegen, putzen' (Id. 9: 2148). Vgl. ahd. swarb m. 'Strudel'; Grimm bringt nhd. Schwerb, ohne dabei auf die nähere Bedeutung einzugehen (Grimm 1899: 9/2588); got. swairban 'wischen'; idg. *suerbh- 'drehen, wischen, fegen' (Pokorny 1: 1050f.).

Die Bedeutung des Worts in unserem Kontext bezieht sich offensichtlich auf die strudelnde und wirbelnde Bewegung von gedämmtem und umgeleitetem Wasser. ${ }^{66}$

\section{Tessel w.}

'Kerbholz mit eingeritzten Namen und Zeichen des Gemeindebürgers'. Die Tesseln bezeichneten die Teilhabe einer Familie an den Gemeinderechten und -pflichten (Id. 4: 282). Es handelte sich gleichsam um "hölzerne Dokumente" (Rütimeyer 1916: 9), die aber auch zu Pflichtaufgebot und Pflichtkontrolle dienten. Sie hingen aufgereiht an einer Schnur im Gemeindehaus. Dem Gegenstand haftet nach Ansicht damaliger Autoren auffälliger Reliktcharakter

66 In einer Regelung von 1548 in Kippel/Lötschen werden die Benutzer dazu aufgefordert Schwerben zu machen, damit sie im Winter auch Wasser haben. 
an. ${ }^{67} \mathrm{Im}$ Wallis sind zahlreiche spezifische Tesseln bezeugt, Weintesseln, Alprechttesseln, Korn- und Weizentesseln; so auch Wassertesseln, auf denen die Wasserrechte eingetragen sind. An manchen Orten hängte man jeweils die Wassertessel desjenigen Bürgers vor das Gemeindehaus, der für die nächsten Stunden sein Land wässern durfte.

Mhd. tassel, tessiel f. 'Pflöcklein oder Leiste zum Zusammenfügen, Spange'. Vgl. afrz. tassel 'Knopf', nfr. Tasseau 'Bindelatte'. Eine sichere Etymologie für das Wort kann noch nicht geleistet werden.

Laut Eichenberger (1940: 94 und Anm.1) war der Gegenstand in Chermignon bis 1933, in Mund, Zeneggen und Lötschen noch in den 1920er Jahren im Gebrauch.

\section{Tretschbord n.}

'Terrassenmauer aus Steinen und Rasenziegeln'. Bielander 1941/42: 91f.: "in schiefer Lage wird auf einen Stein ein Wase (=Rasenziegel) gelegt, auf diesen wieder ein Stein u.s.f."

Zum Bestimmungswort: wallis. Tretscha 'Zopf, Flechte' aus dem ital. treccia (Wipf 1910: 29); vgl. wallis. tretschun, tretschu '(Zöpfe) flechten' (Grichting 1999: 197). Das Wort bezieht sich in unserem Fall auf die besondere Mauertechnik, das Aufeinanderlegen von Steinen und Rasenstücken in je wechselnder Richtung.

Zum Grundwort: wallis. Boord n. 'Bord, Grenzrand', schwzdt. Bord 'Rand, begrenzender Abhang, Böschung', ahd./mhd. bort m. 'Rand, Bord'. In unserem Fall ist die begrenzende Stützmauer am Rand der Wasserleitung gemeint. Sie beugt, so Bielander, Erdrutschen vor (Bielander 1941/42: 91f.)

\section{Wase m.}

'Rasenstück als Stopfmaterial, Bordauffüllung oder Dämmwerk' (Bielander 1941: 95). Wallis. 'Rasenstück' (Grichting 1999: 237). Ahd. waso 'feuchter Rasen', mhd. wase 'Rasen'. Das Wort geht vermutlich auf dieselbe Wurzel zurück wie Rasen, vielleicht auf ein ursprüngliches germ. *wrason (Kluge 1989: 778). Auch: dr Pschöib, siehe bscheibe (Kap. 8).

\section{Wässrblatta $\mathbf{f}$.}

'Portables Eisenblech oder Steinplatte, um die Zuleitung zu dämmen und so das Wasser in die Einlaufrinne zu leiten' (Bielander 1941/42: 101). Das Blech ist unten etwas abgerundet, oben bietet ein eingesägter Griff oder ein kurzer Stiel Halt, um es mitzutragen oder in den Graben zu schieben; (Eichenberger 1940: 91). Ahd. blatta, mhd. plate 'Platte, Steinplatte'.

Gemäss Eichenberger sind die hölzernen Varianten, die Wässerbretter, seltener als die Eisenplatten (Eichenberger 1940: ibid.).

\section{Wässerbiel n.}

'Wässerbeil', siehe Sapa. Zum Grundwort: Ahd. bihal, mhd. bi(h)el, bil 'Spitzhacke, Steinhaue'. Im Wallis. und Schwzdt. kennt man oft nur noch die Diminution: Wallis. Bieli, Bielti 'Beil, Axt' (Grichting 1999: 36).

\section{Wasserrad mit Hammer}

'Signalanlage, die das Fliessen des Wassers mit Klopfgeräuschen anzeigt.'

Vielerorts verschwunden, so in den 1930er Jahren in Zeneggen, Törbel und Visperterminen (Eichenberger 1940: 95). In Ausserberg Dialektausdruck $d r$ Schlegl. Inzwischen werden

\footnotetext{
${ }^{67}$ Schmid führt die eingekerbten Zeichen der Tesseln auf "altgermanische Runenschrift" zurück (Niederer 1993: 45), Rütimeyer bringt gar paläolithische Kerbknochen zum Vergleich (Rütimeyer 1916: 15). Frühes Standardwerk zu den Tesseln im Wallis ist Gmür (1917).
} 
Wasserräder mit Hämmern im Zuge nostalgischer Rückbesinnung oder touristischer Nutzung gelegentlich wieder in Stand gestellt.

\section{Zettstei m.}

'Wässerstein' (Eichenberger 1940: 92). Zum Bestimmungswort siehe Zettwasser (s. Kap. 5.4.1).

\section{Benennung von Tätigkeiten}

\section{abschla}

'die Dämme aus den $\mathrm{Zu}$ - und Hauptleitungen entfernen, damit das Wasser wieder seinen geraden Gang geht'. Abgeschlagen wird, sobald alle Wiesen bewässert worden sind (Eichenberger 1940: 87). Zur Etymologie vgl. die Verbalabstrakta Aschlag und Abschlag (s. Kap. 7).

- ewägg abschlaa (Eichenberger, 1940, 87)

- zr Rü̈̈s schla Rüüs' Hauptwasserleite (s. Kap. 5.1.5.1) (Eichenberger, 1940: 87)

\section{bscheibe}

'Zustopfen von durchlässigen Stellen um die Wässerplatte herum' (Bielander 1941/42: 101f.). Aber auch: 'Dämmen der Zuleitung, um die Felder zu wässern' (In-Albon 1997: 32). Man verwendet Lumpen, alte Kleiderreste, Erde oder Rasenziegel. Wallis. bscheibe 'zustopfen' (Grichting 1999: 43), walser. 'zuschliessen, verkorken' (Szadrowsky 1925: 28), schwzdt. bschäube, bschäibe 'eine Flasche, ein Gefäss, ein Loch, eine Ritze verstopfen' (Id. 8: 73). Ahd. skioban, skiuban, mhd. scieben ,schieben, stossen'.

\section{firschlaa}

'den Bewässerungsdamm (Wässerplatte, Gufr usw.) entfernen und bei dem nächsten zu bewässernden Feld wieder einsetzen, d. h. die Bewässerung um ein Feld vorrücken.' Wallis. vir ' vor 'voraus, voran' (Grichting 1998: 231), wallis. schlaa 'schlagen' (Grichting 1998: 171); vgl. Abschlaga (s. Kap. 7) und abschla (s. Kap. 8).

In Eggerberg vire schla ('nach vorne schlagen') (Bielander 1941/42: 103; In-Albon 1997: 32).

\section{gmeiwärche}

'öffentliche Arbeit der Geteilen verrichten (z. B. die Wasserleitung reinigen)'. Wallis. wärche 'werken' (Grichting 1998: 235), ahd. wirkjan, wirkean, werkan, mhd. wirken 'arbeiten, tätig sein, etw. ins Werk setzen'.

Schwzdt. Gmeiwärch f. (Id. 4: 996), mhd. gemeinwerc 'öffentliche Arbeit' Entlebuch Gmeinwärch 'ungeteilter Grundbesitz einer Gemeinde = Gemeinland' (Waser 2002: 60). Schwzdt. Gmeind f., ahd. gimeinida, mhd. gemeinde 'Gemeinschaft, Gemeinde'. Vgl. zum Gemeinwerk im Wallis das Standardwerk Niederer 1956.

\section{ireise, üsreise}

'Eine Zuleitung dämmen, um das Wasser auf die Wiese zu leiten'. Man verwendet dazu Schieferplatten, zuweilen aber auch hölzerne Wässerbretter, eiserne Platten, Grasstücke, Stoffreste usw. Das Wasser wird entweder in Bewässerungsrinnen eingeleitet oder fassungslos über die Wiese geschwemmt (Eichenberger 1940: 85; Bielander 1941/42: 83/89). Schwzdt. reise 'mit Zielangabe oder der Vorstellung der Richtung einer Sache oder Person (in unserem Fall dem Wasser) eine bestimmte Richtung geben, leiten, lenken, weisen' (Id. 6: 1311). 'Ahd. reison 'aufbrechen, lenken, ordnen mhd. reisen 'bereiten, reisen'. 


\section{izettu}

Synonym zu ireise (Eichenberger 1940: 86; mündliche Mitteilung Reinhard Walter, Grächen, 12.2001).

$\mathrm{Zu}$ Zet- und zeten (s. Kap 5.4.1) Wallis. zeten '(Heu) zerstreuen, ausbreiten'. In unserem Fall also: 'verteilen des Wassers über das Feld'.

\section{zr Zetti üsschlaa}

Synonym zu ireise (Eichenberger 1940: 86). Zetti steht hier wohl für Zetwasserleite/Zuleitung (s. Kap. 5.2.1). Zu üsschlaa vgl. Aschlag (s. Kap 5.4.1).

\section{Wässerturnus m.}

'die festgelegte wiederkehrende Abfolge der Bewässerungen'. (Bielander 1941/42: 96, 98, 100).

Auch: dr Turre, Pl. di Turna (Bielander 1941/42: 96). Mlat. turnus 'Wechsel, Reihenfolge' aus lat. tornus 'Dreheisen, Drechseleisen' und dieses aus gr. tornos 'Zirkel, Dreheisen, Achse' (Kluge 1995: 842). Auch: dr Cheer, siehe Folgendes.

\section{d' Zetti cheeru/ds Wasser cheeru}

Synonym zu firschlaa. Zetti siehe zr Zetti üsschlaa. Walliserdt. cheere 'drehen, wenden, hacken' (Grichting 1999: 47). Ahd. cherjan, keran, mhd. keren 'eine Richtung, Wendung, Umwendung geben'.

$S$ Wasser cheere könnte bedeuten ,die Richtung des Wassers wenden, nämlich vom Schrapf weg wieder entlang des Hauptkanals führen hin zum nächsten Feld'. Vgl. wallis. Cheer m. 'Kurve, Wende, Turnus, Wasserwechsel' (Grichting 1999: ibid.). Wenn der letzte Geteile sein Land bewässert hat, beginnt ein neuer Cheer (Eichenberger 1940: 86).

\section{Zusammenfassung}

\subsection{Epilog}

Wir kehren noch einmal zum Anfang unserer Untersuchung zurück: Zur Zeit, als die ersten Publikationen über die in steilen Felswänden hängenden Suonen erschienen, wurden mithilfe von Sprengstoff und Bohrmaschinen die frühesten Wassertunnels im Wallis realisiert, so etwa in Visperterminen zwischen 1898 und 1916. Man sprengte ganze Felspartien aus, um Bahnen für die Wasserleitungen zu erschliessen (Rauchenstein 1907: 55-61). Bald wurden neben den Holzleitungen auch Kännel aus Eisenblech, Eternit und Zement verlegt (Eichenberger 1940: 5 und 59). Es verbreiteten sich neue wirksame Bewässerungsmethoden, zu denen Pumpsysteme und Sprinkler gehörten. Noch in den jüngsten Jahren sind alte Wasserleitungen ausser Gebrauch genommen worden, so 1992 die Mittla ('die Mittlere') ob Raron (Schmid 1994: 102).

Die Modernisierungen durch Industrie und Technik gingen in ganz Europa einher mit einer wachsenden Aufmerksamkeit für Reliktzonen, die bruchstückhaft eine ländliche Kultur überlieferten. In diesem Zusammenhang gewannen auch die Walliser Wasserleitungen Symbolcharakter für eine massgerechte und überschaubare Wirtschafts- und Lebensweise.

Heute werden im Kontext des Tourismus Bewässerungskanäle oft mit viel Aufwand wieder hergestellt. In den 1970er Jahren in PVC-Rohre verlegte Strecken versetzt man wieder in offene Wasserläufe zurück; Zementabdeckungen weichen Brücken aus Holz. Einer Generation, die selbst noch in den Wasserleitungen arbeitete, erscheint die künstliche Erhaltung von Wasserleitungen dagegen suspekt. Als einer der zwei Verfasser, Werner Bellwald, während der Arbeit an diesem Aufsatz den bebilderten Band Die Suonen des Wallis bei einem Besuch 
seinen Eltern zeigte und fragte, ob sie das Buch auch anschauen möchten, sagte seine Mutter (*1929): "Nimm das Buäch numman bid tiär! Miär pchennän d Siänä va hiä. Miär hein gnuäg drin miässen" ('Nimm das Buch nur wieder mit. Wir kennen die Suonen aus eigener Erfahrung. Wir mussten ja oft genug an ihnen arbeiten').

\subsection{Resultate}

Nicht die Inventarisierung der Namen war Ziel unserer Arbeit. Auch wurden die wenigen Anlagen ausser Acht gelassen, die nicht von Bächen sondern von Weihern (wallis. wier) gespeist werden. ${ }^{68}$

Ein Vergleich mit den Wasserleitungs-Appellativa im übrigen deutschen Sprachraum (Tirol, Elsass, BE, SG, GR) wurde nur am Rande gebracht.

Folgendes konnte in unserer Arbeit geleistet werden:

Wir unterteilten das Oberwallis in ganz, teilweise und nicht bewässerte Zonen. Für das oft als suonenfreies Gebiet dargestellte obere Goms fanden wir Belege und Informanten, die Bewässerungskanäle bezeugen.

Weil die Angaben in der Literatur divergieren, versuchten wir mittels einer Umfrage die sprachgeographische Verteilung der Appellativa Suon und Wasserleite zu erfassen. Die Appllativa zur Bezeichnung von Bewässerungskanälen (Haupt- und Nebenleitungen) wurden gesammelt und ihren Lebendbelegen und historischen Belegen nachgegangen. Bedeutung und Etymologie von jedem Appellativum wurde untersucht.

Von den Eigennamen der Wasserleiten wurde eine erste Typologie und Morphologie erstellt (Kapitel 6).

Im letzten Teil wurden die Benennungen von Konstruktionen, Elementen und Werkzeugen sowie von Tätigkeiten erfasst und ihren Bedeutungen und Etymologien nachgegangen (Kapitel 7 und 8).

\section{Literaturangaben}

Ammann, Hans Robert (1995): "Aperçu sur les documents relatifs aux canaux d'irrigation du Haut-Valais à l'époque médiévale". In: Société d'histoire du Valais romand (ed.): Annales valaisannes. Bulletin annuel de la societé d'histoire du Valais romand. $2^{e}$ série. $70^{e}$ année. Actes du colloque international sur les bisses, Sion 15. - 18. septembre 1994. Sitten: 263280.

Bielander, Josef (1941/42): "Die Bewässerung des Gebietes von Lax". Schweizerisches Archiv für Volkskunde 39: 79-128.

Bohnenberger, Karl (1913): Die Mundart der deutschen Walliser im Heimattal und in den Aussenorten. Frauenfeld. (= Beiträge zur Schweizerdeutschen Grammatik 6).

Braune, Wilhelm (1967): Althochdeutsche Grammatik. Fortgef. v. Karl Helm. Bearb. v. Walther Mitzka. Tübingen.

Bundi, Martin (2000): Zur Geschichte der Flurbewässerung im rätischen Alpengebiet. Chur.

Carlen, Albert (2001): "Ein ertragreicher, ökologisch vielseitiger Lebensraum". In: FlückigerSeiler, Roland/Imhof, Georg/Carlen, Albert (eds.): Bergbauern, Säumer und Durchreisende. Visp: 53-84. (= Erner Schriften: Aus der Vergangenheit für die Zukunft 2).

Crettaz, Bernard (1995): "Autour du bisse: Pour une problématique globale". Annales valaisannes 70: 17-32.

68 Für Mund sind neun solche Weiher bezeugt (Jossen, Mund, 1989, 139f.), für Zeneggen 20, für Törbel 16 (Stebler 1922: 69f.) und einige für Bürchen (z. B. der nach einem Weiler benannte Nässlwier; Oswald Zenhäusern *1932, mündl. Mitt. 02.2002). 
Deutsches Wörterbuch von Jacob Grimm und Wilhelm Grimm (1854-1960). 16 Bde. Leipzig.

Egli, Alfred (1982): Weinbau im Deutschwallis: Sachkultur - Wortschatz - Sprachgeographie. Frauenfeld.

Eichenberger, Ewald (1940): Beitrag zur Terminologie der Walliser "bisses". Diss. Universität Zürich. Aarau.

Furrer, Sigismund (ed.) (1850-1852): Geschichte, Statistik und Urkunden-Sammlung über das Wallis. Sitten.

Pfeiffer, Wolfgang et al. (1989): Etymologisches Wörterbuch des Deutschen. Erarbeitet von einem Autorenkollektiv des Zentralinstituts für Sprachwissenschaft unter der Leitung von Wolfgang Pfeiffer. 3 Bde. Berlin.

FLNB = Liechtensteiner Namenbuch. Die Orts- und Flurnamen des Fürstentums Liechtenstein (1999). Leitung: Hans Stricker. 6 Bde. Vaduz.

Gastinfo Ferienort Saas-Grund (Sommer 2001) 7/4. Jahrgang.

Glatthard, Peter (1987): "Vox alemannica - voces romanicae: Etymologische Miszellen zur Walliser Sprachlandschaft". In: Lüdi, Georges/Stricker, Hans/Wüest, Jakob (eds.): Romania ingeniosa. Festschrift für Prof. Dr. Gerold Hilty zum 60. Geburtstag. Bern und Frankfurt am Main: 3-24.

Gmür, Max (1917): Schweizerische Bauernmarken und Holzurkunden. Mit 33 Tafeln. Bern.

Graff, Eberhard Gottlieb (1834-46): Althochdeutscher Sprachschatz oder Wörterbuch der althochdeutschen Sprache. 6 Teile mit Index v. H. F. Massmann in 3 Bde. Berlin.

Grichting, Alois (1999): Wallissertitschi Weerter: Walliser Wörterbuch. Bd. 1. Visp.

Heer, Jakob-Christoph (1977): An heiligen Wassern: Roman aus dem Wallis. Mit einem Geleitwort v. David Meili. Wetzikon.

Henzen, Walter (1957): Deutsche Wortbildung. Tübingen. (= Sammlung kurzer Grammatiken germanischer Dialekte. Ergänzungsreihe 5).

Hotzenköcherle, Rudolf (1984): Die Sprachlandschaften der deutschen Schweiz. Ed. v. Niklaus Bigler und Robert Schläpfer unter Mitarbeit von Rolf Börlin. Aarau.

Hotzenköcherle, Rudolf (1986): Dialektstrukturen im Wandel: Gesammelte Aufsätze zur Dialektologie der deutschen Schweiz und der Walsergebiete Oberitaliens. Ed. v. Robert Schläpfer und Rudolf Trüb. Aarau.

Homer (1920): Ilias. Übersetzt von Thassilo von Scheffer. Berlin.

Hubschmied, Johannes Ulrich (1938): "Sprachliche Zeugen für das späte Aussterben des Gallischen". Vox Romanica 3/1: 48-155.

Hudry, Marius (1985): "Documents pour une histoire de l'irrigarion en Tarantaise". Le monde alpin et rhodanien 4/1985: 113-120.

Id. = Schweizerisches Idiotikon: Wörterbuch der schweizerdeutschen Sprache (1881-). Bearbeitet von Friedrich Staub, Ludwig Tobler et al. Frauenfeld.

Imseng, Werner (1993): "Volkskundliche und geschichtliche Notizen über die Landwirtschaft von Saas-Fee". In: Supersaxo, Otto et al. (eds.): Saas-Fee: Natur, Landschaft, Landwirtschaft: 10 Jahre Interessenverein Landwirtschaft Saas-Fee. Jubiläumsschrift 1983-1993. Saas Fee: 57-61.

In-Albon, Karl (1997): Die alten Wasserleitungen und Wasserrechte von Eggerberg. Unveröffentlichtes Manuskript. Brig.

Jossen, Erwin (1989): Mund: Das Safrandorf im Wallis. Naters.

Kluge, Friedrich (1995): Etymologisches Wörterbuch der deutschen Sprache. Bearb. v. Elmar Seebold. Berlin/New York.

Köbler, Gerhard (1993): Wörterbuch des althochdeutschen Sprachschatzes. Paderborn/ München/Wien/Zürich.

Lexer, Matthias (1992): Mittelhochdeutsches Handwörterbuch. Stuttgart.

Mathier, Manfred (1989): Die Orts- und Flurnamen der Gemeinde Salgesch im Wallis. Lizentiatsarbeit. Universität Freiburg i. Ü. 
Mariétan, Ignace (1948): Heilige Wasser. Übersetzt von Walter Laedrach. Bern. (= Schweizer Heimatbücher 21/22).

REW = Meyer-Lübke, Wilhelm (1979): Romanisch etymologisches Wörterbuch . Heidelberg.

Niederer, Arnold (1956): Gemeinwerk im Wallis. Bäuerliche Gemeinschaftsarbeit in Vergangenheit und Gegenwart. Diss. Basel. (= Schriften der Schweizerischen Gesellschaft für Volkskunde 37).

Niederer, Arnold (1993): Alpine Alltagskultur zwischen Beharrung und Wandel: Ausgewählte Arbeiten aus den Jahren 1956 - 1991. Bern.

ONB = Ortsnamenbuch des Kantons Bern. Dokumentation und Deutung (1976). Ed. v. Paul Zinsli in Zusammenarbeit mit Rudolf Ramseyer und Peter Glatthard. Bern.

Papilloud, Jean-Henry et al. (eds.) (2000): Die Suonen des Wallis. Visp.

Platter, Thomas (1999): Lebensbeschreibung. Ed. v. Alfred Hartmann. Zweite Auflage, durchgesehen und ergänzt von Ueli Dill. Basel.

Pokorny, Julius (1948-1969): Indogermanisches etymologisches Wörterbuch. 2 Bde. Bern.

Rätisches Namenbuch. Bd. 2: Etymologien (1964). Begründet von Robert von Planta. Bearb. und ed. von Andrea Schorta. Bern.

Rauchenstein, F. (1907): "Die Bewässerungskanäle im Kanton Wallis". Zeitschrift für schweiz. Statistik 44: 52-61.

Reckingen, Dorf und Pfarrei (1995). Ed. von der Gemeinde Reckingen. Visp.

Reynard, Denis (2001): Les Bisses et la gestion de l'irrigation dans le Valais du XVe siècle. Lizentiatsarbeit. Universität Lausanne.

Reynard, Denis (2002): Histoires d'eau: Bisses et irrigation en Valais au XVe siècle. Lausanne.

Rübel, Hans Ulrich (1950): Viehzucht im Oberwallis: Sachkunde, Terminologie, Sprachgeographie. Frauenfeld. (= Beiträge zur schweizerdeutschen Mundartforschung 2).

Rütimeyer, Leopold (1916): Über einige archaistische Gerätschaften und Gebräuche im Kanton Wallis. Basel.

Schade, Oscar (1866): Altdeutsches Wörterbuch. Halle.

Scheuermeier, Paul (1920): Einige Bezeichnungen für den Begriff Höhle in den romanischen Alpendialekten (Balma, Spelunca, Crypta, Tana, Cubulum). Ein wortgeschichtlicher Beitrag zum Studium der alpinen Geländeausdrücke. Halle/Saale.

Schmeller, Johann Andreas (1966): Bayerisches Wörterbuch. Bearb. v. Karl Fromann. 2. Neudruck der Ausgabe München 1872. Aalen.

Schmid, Felix (1981): Ausserberg und sein Wasser. Visp.

Schmid, Maurus (1994): Wasser, kostbares Nass: Die Wasserleitungen an den "Sonnigen Halden", Joli-, Bietsch-, Baltschieder- und Gredetschtal. Visp.

Schnetz, Joseph (1952): Flurnamenkunde. München. (= Bayrische Heimatforschung).

Schnyder, Theo (1940): "Bewässerungsanlagen im Wallis einst und jetzt". Walliser Volksfreund 5. Juli 1940: 5.

Schwäb. Wb. = Fischer, Hermann (1904-1927): Schwäbisches Wörterbuch. Weitergeführt von Wilhelm Pfleiderer. 6 Bd. Tübingen.

Schwarze, Martin (1986): "Wässermatten im Langental". In: Schwarze, Martin (ed.): Die Erhaltung traditioneller Kulturlandschaften. Ed. von der Schweizerischen Stiftung für Landschaftsschutz und Landschaftspflege. Bern: 26-37.

SDS = Sprachatlas der Deutschen Schweiz (1975). Hsg. von Rudolf Hotzenköcherle. In Zusammenarbeit mit Konrad Lobeck, Robert Schläpfer, Rudolf Trüb und unter Mitwirkung von Paul Zinsli. Bd. 3. Bern.

Seebold, Elmar (1970): Vergleichendes und etymologisches Wörterbuch der germanischen starken Verben. The Hague. (= Janua linguarum. Series practica 85). 
Splett, Jochen (1993): Althochdeutsches Wörterbuch: Analyse der Wortfamilienstrukturen des Althochdeutschen, zugleich Grundlegung einer zukünftigen Strukturgeschichte des deutschen Wortschatzes. 2 Bde. Berlin/New York.

Staub, Friedrich (1877): "Ein schweizerisch-alemannisches Lautgesetz". Die deutschen Mundarten. Zeitschrift für Dichtung, Forschung und Kritik 7: 18-389.

Stebler, Friedrich Gottlieb (1901): Ob den Heidenreben. Zürich.

Stebler, Friedrich Gottlieb (1913): Sonnige Halden am Lötschberg. Zürich.

Stebler, Friedrich Gottlieb (1921): Die Vispertaler Sonnenberge. Bern.

Stucki, Karl (1916): Der Vokalismus der Mundart von Jaun im Kanton Freiburg. Diss. Universität Zürich. Frauenfeld.

Vautier, Auguste (1997): Au pays des bisses. Chapelle-sur-Moudon.

Szadrowsky, Manfred (1925): Walserdeutsch. Chur.

Szadrowsky, Manfred (1933): Abstrakta des Schweizerdeutschen in ihrer Sinnentfaltung. Frauenfeld.

Waser, Erika (2002): "Zeugnisse von Ackerbau in der Viehwirtschaftszone des südlichen Kantons Luzern". In: Ernst, Peter/Hausner, Isolde/Schuster, Elisabeth/Wiesinger, Peter (eds.): Ortsnamen und Siedlungsgeschichte. Akten des Symposiums in Wien vom 28. bis 30. September 2000. Heidelberg.

Wendt, Christoph (2001): "Suone, wie Sühne". FAZ 124, 30. Mai 2001: 15.

Werlen, Iwar (1977): "Das 'Staubsche Gesetz' im Schweizerdeutschen". Zeitschrift für Dialektologie und Linguistik 44: 257-281.

Wipf, Elisa (1910): Die Mundart von Visperterminen im Wallis. Frauenfeld. (= Beiträge zur Schweizerdeutschen Grammatik 2).

Zimmermann, Josef (1968): Die Orts- und Flurnamen des Vispertales im Wallis. Diss. Universität Zürich. Zürich.

Zinsli, Paul (1945): Grund und Grat: Die Bergwelt im Spiegel der schweizerdeutschen Alpenmundarten. Bern.

Zinsli, Paul (1984): Südwalser Namengut: Die deutschen Orts- und Flurnamen der ennetbirgischen Walsersiedlungen in Bosco-Gurin und im Piemont. Bern.

Zinsli, Paul (1976): Walser Volkstum. Frauenfeld. 